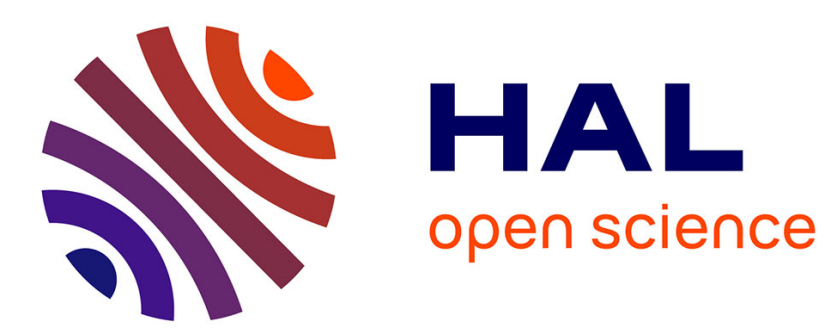

\title{
Languages associated with saturated formations of groups
}

Adolfo Ballester-Bolinches, Jean-Eric Pin, Xaro Soler-Escrivà

\section{To cite this version:}

Adolfo Ballester-Bolinches, Jean-Eric Pin, Xaro Soler-Escrivà. Languages associated with saturated formations of groups. Forum Mathematicum, 2015, Forum Math. 27 (2015), 1471-1505, 27, pp.14711505. 10.1515/forum-2012-0161 . hal-01248004

\section{HAL Id: hal-01248004 https://hal.science/hal-01248004}

Submitted on 23 Dec 2015

HAL is a multi-disciplinary open access archive for the deposit and dissemination of scientific research documents, whether they are published or not. The documents may come from teaching and research institutions in France or abroad, or from public or private research centers.
L'archive ouverte pluridisciplinaire HAL, est destinée au dépôt et à la diffusion de documents scientifiques de niveau recherche, publiés ou non, émanant des établissements d'enseignement et de recherche français ou étrangers, des laboratoires publics ou privés. 


\title{
Languages associated with saturated formations of groups *
}

\author{
Adolfo Ballester-Bolinches, \\ Jean-Éric Pin' ${ }^{2}$ Xaro Soler-Escrivà ${ }^{3}$
}

\begin{abstract}
In a previous paper, the authors have shown that Eilenberg's variety theorem can be extended to more general structures, called formations. In this paper, we give a general method to describe the languages corresponding to saturated formations of groups, which are widely studied in group theory. We recover in this way a number of known results about the languages corresponding to the classes of nilpotent groups, soluble groups and supersoluble groups. Our method also applies to new examples, like the class of groups having a Sylow tower.
\end{abstract}

Warning. By default, all semigroups, monoids, and groups considered in this paper are finite or free. A few exceptions are explicitly stated.

This paper is the second step of a programme aiming at exploring the connections between the formations of finite groups and regular languages.

The first step was to extend Eilenberg's correspondence theorem between varieties of monoids and varieties of languages 9 to the more general setting of formations. The result proved in [3] is quite similar to Eilenberg's theorem: there in a bijective correspondence between formations of finite monoids and the so-called formations of languages. The question is now to effectively describe the languages corresponding to well-studied families of finite groups. Only a few cases have been investigated in the literature:

\footnotetext{
${ }^{1}$ Departament d'Àlgebra, Universitat de València, C/ Dr. Moliner, 50 46100-Burjassot (València), Spain. E-mail: Adolfo.Ballester@uv.es

${ }^{2}$ LIAFA, Université Paris VII and CNRS, Case 7014, 75205 Paris Cedex 13, France.

${ }^{3}$ Dpt. d'Estadística i Investigació Operativa, Universitat d'Alacant, Sant Vicent del Raspeig, Ap. Correus 99, E- 03080 Alacant, Spain.

${ }^{*}$ The authors are supported by Proyecto MTM2010-19938-C03-01 from MICINN (Spain). The first author acknowledges support from MEC. The second author is supported by the project ANR 2010 BLAN 020202 FREC. The third author was supported by the Grant PAID-02-09 from Universitat Politècnica de València.
} 
abelian groups [9], $p$-groups [9, 30, 31, 32], nilpotent groups [9, 28], soluble groups [24, 31] and supersoluble groups [7].

An important step forward would be to find a language theoretic counterpart to the decomposition results in group theory. Indeed, an large part of the research on formations is devoted to the construction of a given formation from simpler ones. For instance, if $\mathbf{F}$ is a group formation, the class $\mathbf{G}_{p} * \mathbf{F}$ of all groups $G$ with a normal $p$-subgroup $N$ such that $G / N \in \mathbf{F}$ is also a formation. This construction plays a crucial role in the study of saturated formations, because the canonical local definition of a saturated formation $\left[8\right.$ is precisely of the form $\mathbf{G}_{p} * \mathbf{F}$.

Coming back to languages, the following question naturally arises:

Given the formation of languages corresponding to $\mathbf{F}$, describe the formation of languages corresponding to $\mathbf{G}_{p} * \mathbf{F}$.

Although this problem was the original motivation of this paper, we were quickly led to a more general question. The point is that, for technical reasons, monoid formations are more suitable than group formations to address this problem. Unfortunately, the notion of normal subgroup does not extend to monoids and it is preferable to replace the operation $\mathbf{G}_{p} * \mathbf{F}$ by a Mal'cev product $\mathbb{L} \mathbf{G}_{p} \mathbb{M} \mathbf{F}$, where $\mathbb{L} \mathbf{G}_{p}$ denotes the class of semigroups which are locally a $p$-group. This trick has been used several times in the study of varieties of monoids [1, 10, 32]. It does not make any change for groups since then $\mathbf{G}_{p} * \mathbf{F}=\mathbb{L} \mathbf{G}_{p} \mathbb{M} \mathbf{F}$ if $\mathbf{F}$ is a group formation, but it gives access to advanced tools of semigroup and automata theory in the general case.

This leads to another problem, in which $\mathbf{F}$ denotes now a formation of monoids:

Given the formation of languages $\mathcal{F}$ corresponding to $\mathbf{F}$, describe the formation of languages $\mathcal{F}^{\prime}$ corresponding to $\mathbb{L} \mathbf{G}_{p}(\mathbb{M}) \mathbf{F}$.

The solution to this problem makes use of the $p$-modular product, an operation on languages first introduced in [23, 24] and widely studied in the literature [1, 7, 10, 11, 27, 30, 31, 32]. The $p$-modular product $\left(L_{0} a_{1} L_{1} \cdots a_{k} L_{k}\right)_{r, p}$ is the set of all words $u$ with $r$ factorizations modulo $p$ of the form $u=$ $u_{0} a_{1} u_{1} \cdots a_{k} u_{k}$ with each $u_{i}$ in $L_{i}$. Our main result can now be stated as follows:

\section{A language belongs to $\mathcal{F}^{\prime}$ if and only if it is a finite Boolean combination of p-modular products of languages of $\mathcal{F}$.}

A similar result was already known for varieties [1, 10, 32. However, the proofs given in these papers rely on properties of the Schützenberger product and the two-sided semidirect product of monoids. Although these notions can be readily extended to varieties of monoids, it is not clear how to extend them to formations. For this reason, we have chosen another road, which 
leads to results of independent interest. The key idea is to decompose $\mathbb{L} \mathbf{G}_{p^{-}}$ morphisms of monoids into irreducible pieces. It follows from the results of [18, 20] that the local monoids of the kernel category of these morphisms are products of cyclic groups of order $p$. Now, if $\pi: M \rightarrow N$ is such an irreducible morphism, we prove the following result:

Every language recognised by $M$ is a finite Boolean combination of languages recognised by $N$ and of p-modular products $\left(L_{0} a L_{1}\right)_{r, p}$, where $L_{0}$ and $L_{1}$ are recognised by $N$.

Then we prove our main result by induction on the number of irreducible pieces in the decomposition of a $\mathbb{L} \mathbf{G}_{p}$-morphism.

Our results allow us to describe the regular languages corresponding to various classes of groups. We first recover as particular cases the known results about languages associated with nilpotent groups, supersoluble groups and soluble groups. Next we treat some new examples, as the languages associated with the class of groups having a Sylow tower. Finally, our result can also be used for formations of monoids. For instance, we describe the languages corresponding to the formation of monoids whose minimal ideal is a $p$-group.

We did our best to keep our paper self-contained, a difficult task since it covers three different areas: semigroup theory, group theory and language theory. With this idea in mind, we tried to organize our material so that a specialist of one of the above-mentioned areas might skip the corresponding section. Accordingly, Section 1 covers various topics of semigroup theory (notably relational morphisms and kernel categories) and Section 2 is devoted to group formations. Section 3 is dedicated to Mal'cev products. The formation theorem is presented in Section 4 and the $p$-modular product is studied in Section 5. Finally, Section 6 contains our main results, Theorem 6.1 and 6.2 .

\section{Background in semigroup theory}

\section{$1.1 \quad$ Semigroups}

An element $e$ of a semigroup is idempotent if $e^{2}=e$. The set of idempotents of a semigroup $S$ is denoted by $E(S)$.

If $e$ is an idempotent of $S$, the set $e S e=\{e s e \mid s \in S\}$ is a monoid with identity $e$, called the local submonoid if $S$ at $e$. A semigroup $S$ is locally a group [p-group] if all of its local submonoids are groups [p-groups].

\subsection{Subdirect products}

Recall that a monoid $M$ is a subdirect product of a family of monoids $\left(M_{i}\right)_{i \in I}$ if $M$ is a submonoid of the direct product $\prod_{i \in I} M_{i}$ and if each induced 
projection $\pi_{i}$ from $M$ onto $M_{i}$ is surjective. The next two propositions relate subdirect products and quotients. We refer to [21, the proof of Lemma 3.2] or to [3, Proposition 1.3]) for the first one and we give a selfcontained proof for the second one.

Proposition 1.1 Let $M$ be a subdirect product of a family of monoids $\left(M_{i}\right)_{i \in I}$. Suppose that, for each $i \in I, M_{i}$ is the quotient of a monoid $T_{i}$. Then $M$ is a quotient of a subdirect product of the family $\left(T_{i}\right)_{i \in I}$.

Let $\left(T_{i}\right)_{i \in I}$ be a family of monoids and, for $i \in I$, let $\mu_{i}: T_{i} \rightarrow N_{i}$ be a surjective monoid morphism. The product of these morphisms is the surjective morphism $\mu: \prod_{i \in I} T_{i} \rightarrow \prod_{i \in I} N_{i}$ defined by $\mu(x)=\left(\mu_{i}(x)\right)_{i \in I}$.

Proposition 1.2 Let $T$ be a subdirect product of the family $\left(T_{i}\right)_{i \in I}$. Then $\mu(T)$ is a subdirect product of the family $\left(N_{i}\right)_{i \in I}$.

Proof. Let $\pi_{i}: \prod_{i \in I} T_{i} \rightarrow T_{i}$ and by $\gamma_{i}: \prod_{i \in I} N_{i} \rightarrow N_{i}$ be the canonical projections. By construction $\gamma_{i} \circ \mu=\mu_{i} \circ \pi_{i}$.

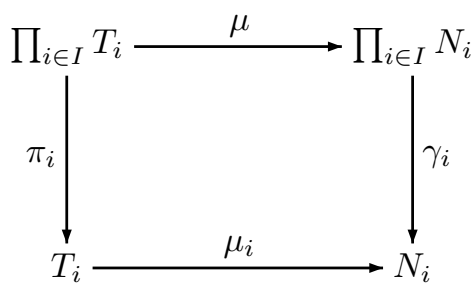

Since $T$ is a subdirect product of the family $\left(T_{i}\right)_{i \in I}$, one has $\pi_{i}(T)=T_{i}$. It follows that $\gamma_{i}(\mu(T))=\mu_{i}\left(\pi_{i}(T)\right)=\mu_{i}\left(T_{i}\right)=N_{i}$. Therefore $\mu(T)$ is a subdirect product of the family $\left(N_{i}\right)_{i \in I}$.

\subsection{Formations and varieties}

A formation of monoids is a class of monoids $\mathbf{F}$ satisfying the two conditions:

(1) any quotient of a monoid of $\mathbf{F}$ also belongs to $\mathbf{F}$,

(2) the subdirect product of any finite family of monoids of $\mathbf{F}$ is also in $\mathbf{F}$.

Formations of semigroups and formations of groups are defined in a similar way. For instance, it is shown in [3] that if $\mathbf{F}$ is a formation of groups, then the monoids whose minimal ideal is a group of $\mathbf{F}$ constitute a formation of monoids, denoted by $\mathbb{E} \mathbf{F}$. In particular, the class $\mathbf{Z}$ of monoids having a zero is a formation of monoids.

A variety of semigroups is a class of semigroups $\mathbf{V}$ satisfying the three conditions:

(1) any subsemigroup of a semigroup of $\mathbf{V}$ also belongs to $\mathbf{V}$,

(2) any quotient of a semigroup of $\mathbf{V}$ also belongs to $\mathbf{V}$, 
(3) the direct product of any finite family of semigroups of $\mathbf{V}$ is also in $\mathbf{V}$. Varieties of monoids [groups] are defined analogously.

It follows from the definition that a formation of semigroups [monoids, groups] is a variety if and only if it is closed under taking subsemigroups [submonoids, subgroups]. Therefore a formation is not necessarily a variety. For instance, the formation of groups generated by the alternating group $A_{5}$ is known to be the class of all direct products of copies of $A_{5}$, which is not a variety [8, II.2.13]. It is also shown in [3] that if $\mathbf{F}$ is a formation of groups, then $\mathbb{I F}$ is a formation of monoids, but is not in general a variety.

If $\mathbf{C}$ is a class of monoids, we denote by $\mathbb{L} \mathbf{C}$ the class of all semigroups whose local submonoids are in $\mathbf{C}$.

\section{Proposition 1.3}

(1) If $\mathbf{V}$ is a variety of monoids, then $\mathbb{L} \mathbf{V}$ is a variety of semigroups.

(2) If $\mathbf{F}$ is a formation of monoids, then $\mathbb{L} \mathbf{F}$ is a formation of semigroups.

Proof. The first part of the proposition is a classical result [9, 13]. Let us prove the second part.

Let $S \in \mathbb{L} \mathbf{F}$ and let $\pi: S \rightarrow T$ be a surjective morphism. Let $f$ be an idempotent of $T$ and let $e$ be an idempotent of $S$ such that $\pi(e)=f$. Then $e S e$ belongs to $\mathbf{F}$ and since $\pi(e S e)=f T f$, the monoid $f T f$ also belongs to $\mathbf{F}$. It follows that $\mathbb{L} \mathbf{F}$ is closed under quotient.

Let now $S$ be a subdirect product of a family $\left(S_{i}\right)_{1 \leqslant i \leqslant n}$ of semigroups of $\mathbb{L} \mathbf{F}$ and let $e$ be an idempotent of $S$. Since $S$ is a subsemigroup of the product $S_{1} \times \cdots \times S_{n}$, one has $e=\left(e_{1}, \ldots, e_{n}\right)$ for some idempotents $e_{1} \in S_{1}$, $\ldots, e_{n} \in S_{n}$. Let $\pi_{i}: S \rightarrow S_{i}$ be the $i$-th projection, which is surjective, since $S$ is a subdirect product. Then $\pi_{i}(e)=e_{i}$ and $\pi_{i}(e S e)=e_{i} S_{i} e_{i}$. It follows that $e S e$ is a subdirect product of the monoids $e_{i} S_{i} e_{i}$. But since $S_{i}$ is in $\mathbb{L} \mathbf{F}$, each monoid $e_{i} S_{i} e_{i}$ belongs to $\mathbf{F}$ and thus $e S e$ belongs to $\mathbf{F}$. Therefore $S$ belongs to $\mathbb{L} \mathbf{F}$, which concludes the proof.

For instance, if $\mathbf{G}$ is the variety of all groups, then $\mathbb{L} \mathbf{G}$ is the variety of all semigroups whose local submonoids are groups. Similarly, given a prime $p$, we denote by $\mathbf{G}_{p}$ the variety of all $p$-groups and by $\mathbb{L} \mathbf{G}_{p}$ the variety of all semigroups whose local submonoids are $p$-groups.

\subsection{Relational morphisms}

Given a monoid $N$, the set of subsets of $N$, denoted $\mathcal{P}(N)$, is a monoid under the multiplication defined, for all $X, Y \subseteq N$, by

$$
X Y=\{x y \mid x \in X, y \in Y\}
$$

A relational morphism between two monoids $M$ and $N$ is a function $\tau$ from $M$ into $\mathcal{P}(N)$ such that: 
(1) for all $m \in M, \tau(m) \neq \emptyset$,

(2) $1 \in \tau(1)$

(3) for all $m, n \in M, \tau(m) \tau(n) \subseteq \tau(m n)$

The composition of two relational morphisms is a relational morphism. Morphisms and inverses of surjective morphisms are standard examples of relational morphisms. In fact, every relational morphism is the composition of a morphism and the inverse of a surjective morphism (See [13, p. 67]).

Proposition 1.4 Let $\tau: M \rightarrow N$ be a relational morphism. Then the set $R=\{(m, n) \in M \times N \mid n \in \tau(m)\}$ is a submonoid of $M \times N$ and the projections from $M \times N$ onto $M$ and $N$ induce morphisms $\alpha: R \rightarrow M$ and $\beta: R \rightarrow N$ such that $\alpha$ is surjective and $\tau=\beta \circ \alpha^{-1}$.

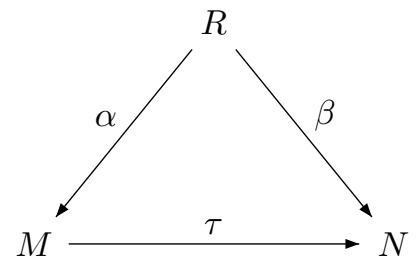

The factorization of $\tau$ given in Proposition 1.4 is called the canonical factorization of $\tau$.

\subsection{Kernel categories}

The kernel of a group morphism is a central notion of group theory. The corresponding notion for monoid morphisms is more difficult to handle and requires the use of categories. We refer to [19, 18, for more details on this topic.

Let $\pi: M \rightarrow N$ be a monoid morphism. Then $M$ acts naturally on $N$ on the right and on the left by setting, for all $m \in M$ and $n \in N$,

$$
m n=\pi(m) n \quad \text { and } \quad n m=n \pi(m) .
$$

Let us consider the category $C_{\pi}$ whose objects are the pairs $\left(n_{0}, n_{1}\right)$ with $\left(n_{0}, n_{1}\right) \in N \times N$ and whose arrows are of the form

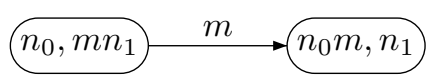

where $m \in M$. Thus $m$ acts by right multiplication on the first component and acts "backwards" by left multiplication on the second component. Composition of arrows is obtained by multiplying their labels: 


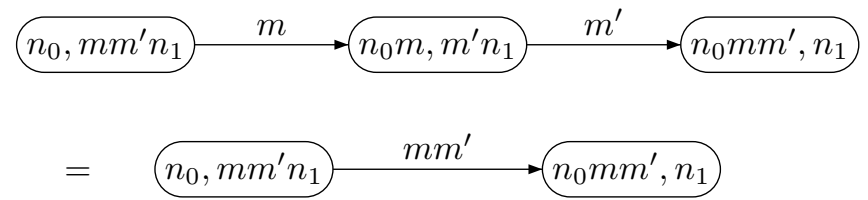

Two arrows

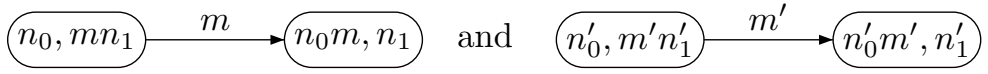

are coterminal if they have same origin and same end, that is, if $n_{0}=n_{0}^{\prime}$, $n_{1}=n_{1}^{\prime}, n_{0} m=n_{0} m^{\prime}$ and $m n_{1}=m^{\prime} n_{1}$.

The kernel category of $\pi$, denoted $\operatorname{ker}(\pi)$, is the quotient of $C_{\pi}$ by the following congruence: two coterminal arrows

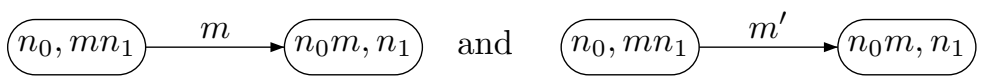

are equivalent if, for all $m_{0} \in \pi^{-1}\left(n_{0}\right)$ and $m_{1} \in \pi^{-1}\left(n_{1}\right)$, one has $m_{0} m m_{1}=$ $m_{0} m^{\prime} m_{1}$.

There is a similar notion for relational morphisms. Let $\tau: M \rightarrow N$ be a relational morphism. We define a category $C_{\tau}$ as follows: its objects are the pairs $\left(n_{0}, n_{1}\right) \in N \times N$ and its arrows are of the form

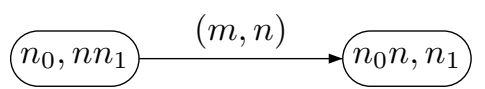

where $n \in \tau(m)$. The kernel category of $\tau$, denoted $\operatorname{ker}(\tau)$, is the quotient of $C_{\tau}$ by the following congruence: two coterminal arrows

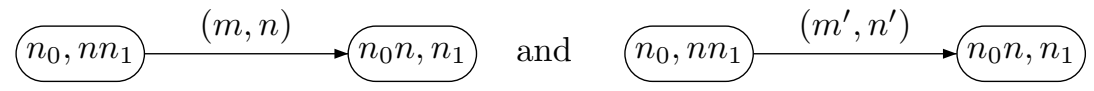

are equivalent if, for all $m_{0} \in \tau^{-1}\left(n_{0}\right)$ and $m_{1} \in \tau^{-1}\left(n_{1}\right)$, one has $m_{0} m m_{1}=$ $m_{0} m^{\prime} m_{1}$.

\section{Group formations}

\section{$2.1 \quad$ Groups}

Let $H, K$ be two normal subgroups of a group $G$ such that $K$ is a subset of $H$. We say that $H / K$ is a chief factor of $G$ if $H / K$ is a minimal normal subgroup of $G / K$. A chief factor $H / K$ of a group $G$ is said to be complemented if there exists a maximal subgroup $M$ of $G$ such that $G=M H$ and $M \cap H=K$. The Frattini subgroup $\Phi(G)$ of a group $G$ is the intersection of all maximal subgroups of $G$. If $H$ is contained in $\Phi(G)$, then the chief factor $H / K$ is 
not complemented. We say in this case that $H / K$ is a Frattini chief factor. Finally, the centraliser of a chief factor $H / K$ in $G$, denoted by $C_{G}(H / K)$, is the set of all $g \in G$ that commute with all elements $h K$ of $H / K$.

Given two classes of groups $\mathbf{V}$ and $\mathbf{W}$, the product $\mathbf{V} * \mathbf{W}$ denotes the class of groups $G$ having a normal subgroup $N \in \mathbf{V}$ such that $G / N \in \mathbf{W}$. If $\mathbf{V}$ and $\mathbf{W}$ are group formations then the product class $\mathbf{V} * \mathbf{W}$ is not in general a formation of groups (see [8, IV, Example (1.6)]). Nevertheless, if $\mathbf{V}$ is closed under taking subnormal subgroups then $\mathbf{V} * \mathbf{W}$ is a formation of groups [8, IV, (1.7)]. In particular, given a formation of groups $\mathbf{W}$ and a prime $p$, the product $\mathbf{G}_{p} * \mathbf{W}$ is always a group formation.

\subsection{Saturated formations}

A formation $\mathbf{F}$ of groups is said to be saturated if $G / \Phi(G) \in \mathbf{F}$ implies $G \in \mathbf{F}$. For instance, the class $\mathbf{N}$ of nilpotent groups is a saturated formation whereas the class $\mathbf{A b}$ of abelian groups is a nonsaturated formation.

A formation function $f$ associates with each prime $p$ a (possibly empty) formation of groups $f(p)$. A formation $\mathbf{F}$ of groups is said to be local if it can be defined locally in the following sense: a group $G$ is in $\mathbf{F}$ if and only if for any complemented chief factor $H / K$ of $G$ and any prime $p$ dividing the order of $H / K$, one has $G / C_{G}(H / K) \in f(p)$. We write $\mathbf{F}=L F(f)$ if $\mathbf{F}$ is locally defined by $f$. For instance, the class $\mathbf{N}$ is a local formation, locally defined by $f(p)=(1)$, for all primes $p$. Indeed, a chief factor $H / K$ of a nilpotent group is always central, that is, $C_{G}(H / K)=G[8$, IV, (3.4)]. Another standard example of a local formation is the class of supersoluble groups. A chief factor of a supersoluble group has always prime order. Thus, the formation of supersoluble groups is locally defined by $f(p)=\mathbf{A} \mathbf{b}(p-1)$, the class of abelian groups of exponent dividing $p-1$, for all primes $p$, IV, (3.4)].

A well-known theorem of group theory states that a formation of groups is saturated if and only if it is local [8, IV, Theorem (4.6)]. In particular, a nonsatured formation cannot be defined locally. For instance, the formation of all abelian groups is not saturated and thus it is not possible to find a local definition for it.

Let $\mathbb{P}$ be the set of all prime numbers. A local formation function $f$ is full if $f(p)=\mathbf{G}_{p} * f(p)$, for all $p \in \mathbb{P}$. Moreover, if $f$ is formation function defining locally a formation $\mathbf{F}$, we say that $f$ is integrated if $f(p) \subseteq \mathbf{F}$ for all $p \in \mathbb{P}$ (see [8, III, (5.5)]).

In general, a saturated formation $\mathbf{F}$ possesses many local definitions but it has a unique full and integrated local formation function [8, IV, (3.7)], which is said to be its canonical local definition. Given a saturated formation $\mathbf{F}$ locally defined by a formation function $f$, the canonical local definition $F$ of $\mathbf{F}$ is defined by $F(p)=\mathbf{G}_{p} *(f(p) \cap \mathbf{F})$, for all $p \in \mathbb{P}[8$, IV, (3.8)]. Thus, the canonical local definition of $\mathbf{N}$ is $\mathbf{G}_{p}$, for all primes $p$ and the canonical 
local definition of the supersoluble groups is $\mathbf{G}_{p} * \mathbf{A b}(p-1)$, for all primes $p$. We refer the reader to [8, IV] for a complete account on this topic.

Denote by $\bigvee_{i \in I} \mathbf{H}_{i}$ the join of a family of formations of groups $\left(\mathbf{H}_{i}\right)_{i \in I}$, that is, the smallest formation which contains $\mathbf{H}_{i}$ for all $i \in I$.

Lemma 2.1 Let $\mathbf{F}$ be a saturated formation of groups and let $F$ be the canonical local definition of $\mathbf{F}$. Then $\mathbf{F}=\bigvee_{p \in \mathbb{P}} F(p)$.

Proof. Let $\mathbf{X}=\bigvee_{p \in \mathbb{P}} F(p)$. Since $F$ is an integrated formation function, one has $F(p) \subseteq \mathbf{F}$ for all primes $p$. Thus $\mathbf{X} \subseteq \mathbf{F}$. Conversely, suppose that $\mathbf{F} \backslash \mathbf{X} \neq \emptyset$ and consider a group $G \in \mathbf{F} \backslash \mathbf{X}$ of minimal order. Then $G$ has a unique minimal normal subgroup, say $N$ [8, II, (2.5)(a)]. Let $p$ be a prime dividing the order of $N$. We know that $\mathbf{F}=\bigcap_{q \in \operatorname{Char}(\mathbf{F})} \mathbf{G}_{q^{\prime}} * F(q)[\underline{8}$, (IV), (3.2)], where $\operatorname{Char}(\mathbf{F})$ is the set of primes $p$ such that $\mathbf{F}$ contains a cyclic group of order $p$ and $\mathbf{G}_{q^{\prime}}$ is the class of groups whose orders are prime to $q$. Since $p$ divides the order of $G \in \mathbf{F}$, it follows that $p \in \operatorname{Char}(\mathbf{F})$ [8, IV, (4.2)]. Thus $G \in \mathbf{G}_{p^{\prime}} * F(p)$. Then $G \in F(p) \subseteq \mathbf{X}$ because $G$ has no nontrivial normal subgroups of $p^{\prime}$-order.

The preceding lemma is not true if the local definition of $\mathbf{F}$ is not the canonical one. For instance, consider the formation of supersoluble groups and its local definition $f(p)=\mathbf{A} \mathbf{b}(p-1)$, for all primes $p$. This is an integrated local definition which is not full. Clearly, the join of the formations $\mathbf{A b}(p-1)$ for all primes $p$ is properly contained in the class of supersoluble groups.

\section{Mal'cev products}

The Mal'cev product is an important tool in the study of varieties of semigroups [16]. We propose in this section an extension of this definition to more general classes than varieties.

\subsection{C-morphisms and $\ell$ C-morphisms}

Let $\mathbf{C}$ be a class of semigroups. A [relational] morphism $\tau: M \rightarrow N$ is said to be a [relational] C-morphism if for every idempotent $e$ of $N$, the semigroup $\tau^{-1}(e)$ belongs to $\mathbf{C}$. The following result is very convenient in practice since it allows one to replace relational $\mathbf{C}$-morphisms by $\mathbf{C}$-morphisms.

Proposition 3.1 Let $M \stackrel{\alpha^{-1}}{\longrightarrow} R \stackrel{\beta}{\longrightarrow} N$ be the canonical factorization of $a$ relational morphism $\tau: M \rightarrow N$. Then $\tau$ is a relational $\mathbf{C}$-morphism if and only if $\beta$ is a $\mathbf{C}$-morphism.

Proof. Let $e$ be an idempotent of $N$. Then by definition,

$$
\tau^{-1}(e)=\{m \in M \mid e \in \tau(m)\} \text { and } \beta^{-1}(e)=\{(m, e) \in M \times N \mid e \in \tau(m)\} .
$$


It follows that $\tau^{-1}(e)$ and $\beta^{-1}(e)$ are isomorphic semigroups. The result follows.

We are mostly interested in [relational] $\mathbb{L} \mathbf{G}_{p}$-morphisms in this paper. They share the same properties as relational $\mathbb{L} \mathbf{G}$-morphisms and the proof of the following result can be readily adapted from the proof of [13, Proposition 5.8 , p. 71].

Proposition 3.2 Let $\tau: M \rightarrow N$ be a relational morphism. Then $\tau$ is a relational $\mathbb{L} \mathbf{G}_{p}$-morphism if and only if for each subsemigroup $S$ of $N$ belonging to $\mathbb{L} \mathbf{G}_{p}$, the semigroup $\tau^{-1}(S)$ also belongs to $\mathbb{L} \mathbf{G}_{p}$. It follows that the composition of two relational $\mathbb{L} \mathbf{G}_{p}$-morphisms is a relational $\mathbb{L} \mathbf{G}_{p^{-}}$ morphism.

If $n$ is an object of a category, the arrows from $n$ to $n$ form a monoid, called the local monoid the category at $n$. Let $\mathbf{C}$ be a class of monoids. A category is said to be locally in $\mathbf{C}$ if its local monoids belong to C. Similarly, a [relational] morphism $\pi: M \rightarrow N$ is said to be a [relational] $\ell \mathbf{C}$-morphism if the category $\operatorname{ker}(\pi)$ is locally in $\mathbf{C}$. The next proposition can be viewed as a counterpart of Proposition 3.1.

Proposition 3.3 Let $M \stackrel{\alpha^{-1}}{\longrightarrow} R \stackrel{\beta}{\longrightarrow} N$ be the canonical factorization of a relational morphism $\tau: M \rightarrow N$. Then $\tau$ is a relational $\ell \mathbf{C}$-morphism if and only if $\beta$ is a $\ell \mathbf{C}$-morphism.

Proof. Let $\left(n_{0}, n_{1}\right) \in N \times N$. By definition, the local monoids of $C_{\beta}$ and $C_{\tau}$ at $\left(n_{0}, n_{1}\right)$ are both equal to

$$
T=\left\{(m, n) \in R \mid n_{0} n=n_{0} \text { and } n n_{1}=n_{1}\right\}
$$

It follows that the local monoids of $\operatorname{ker}(\beta)$ and $\operatorname{ker}(\tau)$ at $\left(n_{0}, n_{1}\right)$ are isomorphic monoids, obtained as the quotient of $T$ by the congruence defined by $m \sim m^{\prime}$ if for all $\left(m_{0}, n_{0}\right),\left(m_{1}, n_{1}\right) \in R, m_{0} m m_{1}=m_{0} m^{\prime} m_{1}$. The result follows.

The next proposition, a slight extension of [1, Proposition 5.3], gives the connection between the notions of $\ell \mathbf{C}$-morphisms and $\mathbb{L} \mathbf{C}$-morphisms.

Proposition 3.4 Let $\mathbf{C}$ be a class of monoids closed under taking submonoids and quotients. Then every [relational] $\ell \mathbf{C}$-morphism is a [relational] $\mathbb{L} \mathbf{C}$-morphism.

Propositions 3.1 and 3.3 show that it suffices to give the proof in the case of morphisms. We need an elementary lemma.

Lemma 3.5 Let $M$ be a monoid. Any subsemigroup of $M$ which is also a monoid is a quotient of a submonoid of $M$. 
Proof. Let $S$ be a subsemigroup of $M$ and suppose that $S$ is a monoid with identity $e$. The subtle point is that $e$ might be different from the identity 1 of $M$, and thus $S$ is not in general a submonoid of $M$. However, $S \cup\{1\}$ is a submonoid of $M$. Further, the map from $S \cup\{1\}$ to $S$ defined by $\pi(1)=e$ and $\pi(s)=s$ if $s \in S$ defines a monoid morphism from $S \cup\{1\}$ onto $S$.

We now come back to the proof of Proposition 3.4. Let us denote by $\sim$ the congruence introduced in the definition of the kernel category of a morphism.

Proof. Let $\pi: M \rightarrow N$ be a $\ell \mathbf{C}$-morphism and let $e$ be an idempotent of $N$. Let $T$ be the local monoid of the kernel category of $\pi$ at $(e, e)$. Since $\pi$ is $\ell \mathbf{C}$-morphism, $T$ is in C. Let $S=\pi^{-1}(e)$ and let $f$ be an idempotent of $S$. We claim that $f S f$ is a subsemigroup of $T$. Let $s$ be an element of $f S f$. Since $e \pi(f s f)=\pi(f s f) e=e e=e$, there is an arrow $(e, e) \stackrel{f s f}{\longrightarrow}(e, e)$. Further, two $\sim$-equivalent arrows, labelled respectively by $f s f$ and $f s^{\prime} f$, are necessarily equal, since, by the definition of $\sim$, one has in particular $f(f s f) f=f\left(f s^{\prime} f\right) f$, that is, $f s f=f s^{\prime} f$. It follows that $f S f$ is a subsemigroup of $T$ and is also a monoid with $f$ as an identity. By Lemma 3.5, the monoid $f S f$ is a quotient of a submonoid of $T$ and thus belongs to $\mathbf{C}$. Consequently, $S$ is in $\mathbb{L} \mathbf{C}$ and $\pi$ is a $\mathbb{L} \mathbf{C}$-morphism.

Example 3.1 below shows that the converse of Proposition 3.4 does not hold, even if $\mathbf{C}$ is the trivial class $\mathbf{1}$ containing only the trivial monoid.

Example 3.1 Let $M=\left\{1, a, a^{2}, 0\right\}$, with $a^{3}=0$ and let $N=\{1,0\}$. Let $\pi: M \rightarrow N$ be the morphism defined by $\pi(1)=1$ and $\pi(a)=\pi\left(a^{2}\right)=$ $\pi(0)=0$. Then $\pi^{-1}(1)=1$ and $\pi^{-1}(0)=\left\{a, a^{2}, 0\right\}$. The unique idempotent of $\pi^{-1}(0)$ is 0 and $0 \pi^{-1}(0) 0=0$. Thus $\pi$ is a $\mathbb{L} \mathbf{1}$-morphism. However, in the category $\operatorname{ker}(\pi)$, the local monoid at $(0,0)$ is nontrivial. Indeed, the two loops of $C_{\pi}$

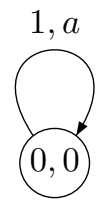

are not equivalent in $\operatorname{ker}(\pi)$ since $a \in \pi^{-1}(0)$, but $a 1 a=a^{2}$ and $a a a=0$. Therefore, $\pi$ is not a $\ell \mathbf{1}$-morphism.

\subsection{The Mal'cev product $\mathrm{C}$ (M) D}

Let $\mathbf{C}$ be class of semigroups and let $\mathbf{D}$ be a class of monoids. The Mal'cev product $\mathbf{C} \otimes \mathbf{D}$ is the class of all monoids $T$ with the following property: $T$ 
is a quotient of a monoid $M$ for which there exists a surjective $\mathbf{C}$-morphism from $M$ onto a monoid $N$ of $\mathbf{D}$.

This is pictured in Figure 3.1, in which a surjective [relational] morphism is represented by a double arrow.

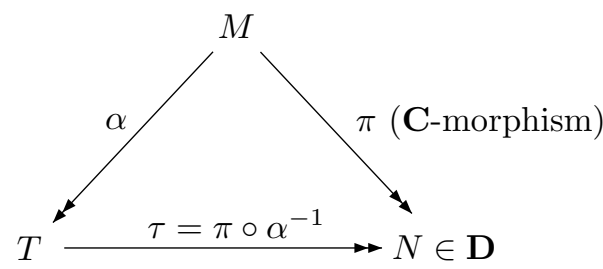

Figure 3.1: A monoid $T$ in $\mathbf{C} \mathbb{M}$.

The next proposition gives an alternate definition of the Mal'cev product $\mathbf{C}(\mathbb{M}) \mathbf{D}$ when $\mathbf{C}$ is closed under quotients.

Proposition 3.6 Let $\mathbf{C}$ be a class of semigroups closed under quotients and let $\mathbf{D}$ be a class of monoids. A monoid $M$ belongs to $\mathbf{C} \mathbb{M}$ if and only if there is a relational $\mathbf{C}$-morphism from $M$ onto a monoid of $\mathbf{D}$.

Proof. Let $M$ be a monoid of $\mathbf{C} \mathbb{A}$ D. Let $\tau$ be the relational morphism defined by Figure 3.1. We claim that $\tau$ is a relational C-morphism. Let $e \in$ $E(N)$ and let $R=\pi^{-1}(e)$. Then $R$ belongs to $\mathbf{C}$ since $\pi$ is a $\mathbf{C}$-morphism. Now, $\tau^{-1}(e)$ is equal to $\alpha(R)$ and thus is a quotient of $R$. Consequently, it also belongs to $\mathbf{C}$, which proves the claim.

Suppose now there is a relational C-morphism $\tau$ from $T$ onto a monoid $N$ of $\mathbf{D}$. Let $T \stackrel{\alpha^{-1}}{\longrightarrow} R \stackrel{\beta}{\longrightarrow} N$ be the canonical factorization of $\tau$. By Proposition 3.1, $\beta$ is a surjective $\mathbf{C}$-morphism and $T$ is a quotient of $R$. Therefore $T$ belongs to $\mathbf{C} \mathbb{( M} \mathbf{D}$.

Proposition 3.7 Let $\mathbf{V}$ be a variety of semigroups. If $\mathbf{W}$ is a formation [variety] of monoids, then so is $\mathbf{V} \mathbb{M} \mathbf{W}$.

Proof. The result is well-known for varieties and thus we give only the proof for formations. Let $\mathbf{F}=\mathbf{V}$ (M) $\mathbf{W}$.

It follows directly from the definition that $\mathbf{F}$ is closed under quotients. Let $M$ be a subdirect product of some monoids $M_{1}, \ldots, M_{r}$ of $\mathbf{F}$. By definition, each $M_{i}$ is a quotient of a monoid $T_{i}$ for which there is a surjective $\mathbf{V}$-morphism $\mu_{i}$ from $T_{i}$ onto a monoid $N_{i}$ of $\mathbf{W}$. It follows from Proposition 1.1 that $M$ is a quotient of a subdirect product $T$ of $T_{1}, \ldots, T_{r}$. Let us take the notation of Proposition 1.2. Let $N=\mu(T)$. Then $N$ is a subdirect product of $N_{1}, \ldots, N_{r}$ and thus $N \in \mathbf{W}$. We claim that $\mu$ is a $\mathbf{V}$-morphism. Let $e=\left(e_{1}, \ldots, e_{r}\right)$ be an idempotent of $N$. Setting $R_{i}=\mu_{i}^{-1}\left(e_{i}\right)$ and 
$R=R_{1} \times \cdots \times R_{r}$, we get $\mu^{-1}(e)=T \cap R$. Now since each $\mu_{i}$ is a $\mathbf{V}$ morphism, each semigroup $R_{i}$ belongs to $\mathbf{V}$ and so does $R$. Finally, $T \cap R$ is a subsemigroup of $R$ and thus also belongs to $\mathbf{V}$. This proves the claim and shows that $M \in \mathbf{F}$. Therefore $\mathbf{F}$ is a formation of monoids.

\subsection{The Mal'cev product $\ell \mathrm{C}(\mathrm{M}) \mathrm{D}$}

The Mal'cev product $\ell \mathbf{C} \mathbb{} \mathbf{D}$ can be defined in a similar way: it is the class of all monoids $T$ with the following property: $T$ is a quotient of a monoid $M$ for which there exists a surjective $\ell \mathbf{C}$-morphism $\pi$ from $M$ onto a monoid of $\mathbf{D}$.

Again, an alternative definition can be given when $\mathbf{C}$ is closed under quotients.

Proposition 3.8 Let $\mathbf{C}$ be a class of semigroups closed under quotients and let $\mathbf{D}$ be a class of monoids. A monoid $M$ belongs to $\ell \mathbf{C}(\mathbb{M} \mathbf{D}$ if and only if there is a relational $\ell \mathbf{C}$-morphism from $M$ onto a monoid of $\mathbf{D}$.

Proof. The proof is similar to that of Proposition 3.6 but makes use of Proposition 3.3 instead of Proposition 3.1.

Proposition 3.9 Let $\mathbf{V}$ be a variety of semigroups. If $\mathbf{W}$ is a formation [variety] of monoids, then so is $\ell \mathbf{V} \mathbb{M} \mathbf{W}$.

Proof. The result is already known for varieties and we prove it only for

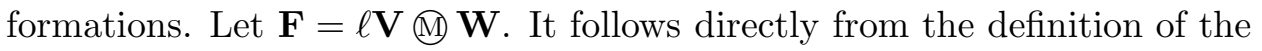
Mal'cev product that $\mathbf{F}$ is closed under quotients. Let $M$ be a subdirect product of a finite family $\left(M_{i}\right)_{i \in I}$ of monoids of $\mathbf{F}$. By definition, each $M_{i}$ is a quotient of a monoid $T_{i}$ for which there is a surjective $\ell \mathbf{V}$-morphism $\mu_{i}$ from $T_{i}$ onto a monoid $N_{i}$ of $\mathbf{W}$. It follows from Proposition 1.1 that $M$ is a quotient of a subdirect product $T$ of the family $\left(T_{i}\right)_{i \in I}$. It suffices now to prove that $T$ belongs to $\mathbf{F}$. Let us take the notation of Proposition 1.2 and let $N=\mu(T)$.

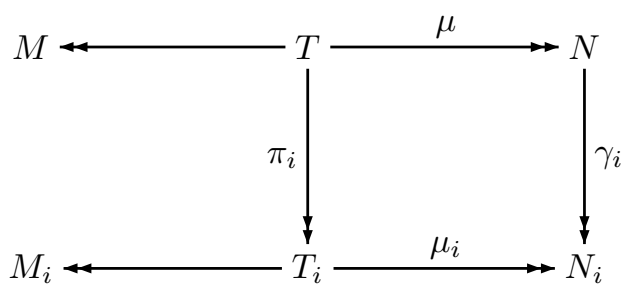

Then $N$ is a subdirect product of the family $\left(N_{i}\right)_{i \in I}$ and thus belongs to $\mathbf{W}$. Therefore, it just remains to show that $\mu$ is a $\ell \mathbf{V}$-morphism to complete the proof. Let $n_{0}=\left(n_{0}\right)_{i \in I}$ and $n_{1}=\left(n_{1}\right)_{i \in I}$ be elements of $N$. Let $\operatorname{Loc}\left(n_{0}, n_{1}\right)$ be the local monoid of $\operatorname{ker}(\mu)$ at $\left(n_{0}, n_{1}\right)$. Similarly, for each $i \in I$, let 
$\operatorname{Loc}_{i}\left(n_{0}, n_{1}\right)$ be the local monoids of $\operatorname{ker}\left(\mu_{i}\right)$ at $\left(\left(n_{0}\right)_{i},\left(n_{1}\right)_{i}\right)$. We need to prove that $\operatorname{Loc}\left(n_{0}, n_{1}\right)$ belongs to $\mathbf{V}$. This will be a consequence of the following lemma:

Lemma 3.10 The semigroup $\operatorname{Loc}\left(n_{0}, n_{1}\right)$ is a subsemigroup of the product of the family $\left(\operatorname{Loc}_{i}\left(n_{0}, n_{1}\right)\right)_{i \in I}$.

Proof. The monoid $\operatorname{Loc}\left(n_{0}, n_{1}\right)$ is the quotient of the monoid

$$
\operatorname{Stab}\left(n_{0}, n_{1}\right)=\left\{t \in T \mid n_{0} \mu(t)=n_{0} \text { and } \mu(t) n_{1}=n_{1}\right\}
$$

by the congruence $\sim$ defined by $t \sim t^{\prime}$ if, for all $t_{0}, t_{1} \in T$ such that $\mu\left(t_{0}\right)=n_{0}$ and $\mu\left(t_{1}\right)=n_{1}$, one has $t_{0} t t_{1}=t_{0} t^{\prime} t_{1}$. In the same way, for each $i \in I$, $\operatorname{Loc}_{i}\left(n_{0}, n_{1}\right)$ is the quotient of the monoid

$$
\operatorname{Stab}_{i}\left(n_{0}, n_{1}\right)=\left\{t_{i} \in T_{i} \mid\left(n_{0}\right)_{i} \mu_{i}\left(t_{i}\right)=\left(n_{0}\right)_{i} \text { and } \mu_{i}\left(t_{i}\right)\left(n_{1}\right)_{i}=\left(n_{1}\right)_{i}\right\}
$$

by the corresponding congruence $\sim_{i}$. Let us denote by $\gamma_{i}$ be the canonical morphism from $\operatorname{Stab}_{i}\left(n_{0}, n_{1}\right)$ to $\operatorname{Loc}_{i}\left(n_{0}, n_{1}\right)$. If $t$ is an element of $\operatorname{Stab}\left(n_{0}, n_{1}\right)$, then $\pi_{i}(t)$ belongs to $\operatorname{Stab}_{i}\left(n_{0}, n_{1}\right)$. Thus $\pi_{i}$ induces a morphism from $\operatorname{Stab}\left(n_{0}, n_{1}\right)$ to $\operatorname{Stab}_{i}\left(n_{0}, n_{1}\right)$. Let $\alpha$ be the morphism from $\operatorname{Stab}\left(n_{0}, n_{1}\right)$ to $\prod_{i \in I} \operatorname{Loc}_{i}\left(n_{0}, n_{1}\right)$ defined by $\alpha(t)=\left(\gamma_{i} \circ \pi_{i}(t)\right)_{i \in I}$. One has $\alpha(t)=\alpha\left(t^{\prime}\right)$ if and only if, for each $i \in I, t_{i} \sim_{i} t_{i}^{\prime}$, that is, $t \sim t^{\prime}$. It follows that $\operatorname{Loc}\left(n_{0}, n_{1}\right)$ is a subsemigroup of $\prod_{i \in I} \operatorname{Loc}_{i}\left(n_{0}, n_{1}\right)$.

Let us now conclude the proof of Proposition 3.9. Since $\mu_{i}$ is a $\ell \mathbf{V}$ morphism, $\operatorname{Loc}_{i}\left(n_{0}, n_{1}\right)$ belongs to $\mathbf{V}$. Since $\mathbf{V}$ is a variety of semigroups, Lemma 3.10 shows that $\operatorname{Loc}\left(n_{0}, n_{1}\right)$ also belongs to $\mathbf{V}$. Therefore $\mu$ is a $\ell \mathbf{V}$-morphism and thus $T$ and $M$ are in $\mathbf{F}$. Consequently, $\mathbf{F}$ is a formation of monoids.

\subsection{Factorisations of morphisms}

A surjective morphism $\pi$ between monoids is said to be irreducible if it is not an isomorphism and if $\pi=\pi_{0} \circ \pi_{1}$, then one of $\pi_{0}$ or $\pi_{1}$ is an isomorphism. By a theorem of Rhodes [17], every surjective morphism between monoids is a composition of irreducible morphisms. Similarly, every surjective $\mathbb{L} \mathbf{G}_{p^{-}}$ morphism between monoids is a composition of irreducible $\mathbb{L} \mathbf{G}_{p}$-morphisms.

Irreducible morphisms have been widely studied and we refer to [20, 18] for an overview. The following result is an easy consequence of [18, Theorem 5.3.2], which itself summarizes the results of [20]. Let $\mathbf{C}_{p}$ be the class consisting of the cyclic group $C_{p}$ and of the trivial group 1.

Proposition 3.11 An irreducible $\mathbb{L} \mathbf{G}_{p}$-morphism is a $\ell \mathbf{C}_{p}$-morphism. 
Proof. Let $\pi: M \rightarrow N$ be an irreducible $\mathbb{L} \mathbf{G}_{p}$-morphism. By [18, Theorem 5.3.2], an irreducible morphism is either $\mathbb{L} \mathbf{G}$ but not aperiodic, aperiodic but not $\mathbb{L} \mathbf{1}$, or $\mathbb{L} \mathbf{1}$. Since $\pi$ is a $\mathbb{L} \mathbf{G}$-morphism and since an aperiodic $\mathbb{L} \mathbf{G}$ morphism is $\mathbb{L} \mathbf{1}$, the second case does not arise. By the same result, an irreducible $\mathbb{L} \mathbf{1}$-morphism is $\ell \mathbf{1}$ and hence also $\ell \mathbf{C}_{p}$. Finally, Property (1) of the same result shows that if $\pi$ is not aperiodic, then for each idempotent $e$ of $N, \pi^{-1}(e)$ is a product of simple $p$-groups. But the only simple $p$-group is $C_{p}$ and thus $\pi$ is an $\ell \mathbf{C}_{p}$-morphism.

Example 3.2 Let us come back on the morphism $\pi$ defined in Example 3.1. For each prime $p, \pi$ is a $\mathbb{L} \mathbf{G}_{p}$-morphism, but it is not a $\ell \mathbf{C}_{p}$-morphism. However, it is the composition of the two irreducible $\ell \mathbf{1}$-morphisms $\pi_{1}$ : $M \rightarrow R$ and $\pi_{2}: R \rightarrow N$ defined as follows: $\pi_{1}(1)=1, \pi_{1}(a)=a$ and $\pi_{1}\left(a^{2}\right)=\pi_{1}(0)=0, \pi_{2}(1)=1$ and $\pi_{2}(a)=\pi_{2}(0)=0$.

\subsection{Mal'cev products of the form $\mathbb{L} \mathbf{G}_{p} \mathbb{M} \mathbf{W}$ and $\ell \mathbf{A b}(p) \mathbb{M} \mathbf{W}$}

In this paper, we are mostly interested in Mal'cev products of the form $\mathbb{L} \mathbf{G}_{p} \mathbb{M} \mathbf{W}$ and $\ell \mathbf{A b}(p) \mathbb{M} \mathbf{W}$. In this case, the definition of the Mal'cev product can be simplified. We shall use the following results of [1]. First of all, for each monoid $M$, there is a largest monoid congruence $\operatorname{Rad}_{p}(M)$ such that the morphism $M \rightarrow M / \operatorname{Rad}_{p}(M)$ is a $\mathbb{L} \mathbf{G}_{p}$-morphism. Further, if $\varphi: M \rightarrow N$ is a surjective morphism of monoids, then $\varphi$ induces a surjective morphism $\bar{\varphi}: M / \operatorname{Rad}_{p}(M) \rightarrow N / \operatorname{Rad}_{p}(N)$. We can now formulate the following extension of [1, Theorem 3.8], the proof of which is unchanged.

Proposition 3.12 Let $\mathbf{W}$ be a formation of monoids and let $M$ be a monoid. The following conditions are equivalent:

(1) $M$ belongs to $\mathbb{L} \mathbf{G}_{p} \mathbb{M} \mathbf{W}$,

(2) there is a surjective $\mathbb{L} \mathbf{G}_{p}$-morphism from $M$ onto a monoid of $\mathbf{W}$,

(3) $M / \operatorname{Rad}_{p}(M) \in \mathbf{W}$.

The next proposition gives the precise connection between $\mathbb{L} \mathbf{G}_{p} \mathbb{M} \mathbf{W}$ and $\ell \mathbf{A b}(p) \mathbb{B} \mathbf{W}$.

Proposition 3.13 The formation $\mathbb{L} \mathbf{G}_{p} \mathbb{M} \mathbf{W}$ is the least formation containing $\mathbf{W}$ and closed under Mal'cev product (on the left) by $\ell \mathbf{A b}(p)$.

Proof. Let $\mathbf{F}$ be the least formation containing $\mathbf{W}$ and closed under Mal'cev product (on the left) by $\ell \mathbf{A b}(p)$.

To prove the inclusion $\mathbf{F} \subseteq \mathbb{L} \mathbf{G}_{p} \mathbb{M} \mathbf{W}$, we show that $\mathbb{L} \mathbf{G}_{p} \mathbb{M} \mathbf{W}$ is closed under Mal'cev product (on the left) by $\ell \mathbf{A b}(p)$. If $T$ belongs to $\ell \mathbf{A b}(p) \mathbb{M}\left(\mathbb{L} \mathbf{G}_{p} \mathbb{M} \mathbf{W}\right)$, there exists a surjective $\ell \mathbf{A b}(p)$-morphism $\gamma: T \rightarrow$ $M$, where $M \in \mathbb{L} \mathbf{G}_{p} \mathbb{M} \mathbf{W}$. Further, by Proposition 3.12, there is a surjective $\mathbb{L} \mathbf{G}_{p}$-morphism $\pi$ from $M$ onto a monoid $N$ of $\mathbf{W}$. By Proposition 3.4, 
$\gamma$ is a $\mathbb{L} \mathbf{A} \mathbf{b}(p)$-morphism and hence a $\mathbb{L} \mathbf{G}_{p}$-morphism. Since the composition of two $\mathbb{L} \mathbf{G}_{p}$-morphisms is a $\mathbb{L} \mathbf{G}_{p}$-morphism by Proposition 3.2, $\pi \circ \gamma$ is a $\mathbb{L} \mathbf{G}_{p}$-morphism from $T$ onto $N$, which shows that $T$ belongs to $\mathbb{L} \mathbf{G}_{p} \mathbb{M} \mathbf{W}$.

To prove the opposite inclusion, consider a monoid $M$ in $\mathbb{L} \mathbf{G}_{p} \mathbb{M} \mathbf{W}$. Then there is a surjective $\mathbb{L} \mathbf{G}_{p}$-morphism $\pi$ from $M$ onto a monoid of $\mathbf{W}$. Let us write $\pi$ as a composition of irreducible morphisms: $M=M_{k} \stackrel{\pi_{k}}{\longrightarrow}$ $M_{k-1} \ldots \stackrel{\pi_{1}}{\longrightarrow} M_{0}$. By Proposition 3.11, each $\pi_{i}$ is a $\ell \mathbf{A b}(p)$-morphism and it follows by induction on $i$ that $M_{i}$ belongs to $\mathbf{F}$. Finally $M$ also belongs to $\mathbf{F}$, which concludes the proof.

Let us conclude this section by two examples.

Proposition 3.14 If $\mathbf{H}$ is a variety of groups and $\mathbf{W}$ is a formation of groups, then $\mathbb{L} \mathbf{H} \mathbb{M} \mathbf{W}=\mathbf{H} * \mathbf{W}$.

Proof. Let $G$ be a group of $\mathbf{H} * \mathbf{W}$. By definition, $G$ has a normal subgroup $N \in \mathbf{H}$ such that $G / N \in \mathbf{W}$. We claim that the morphism $\pi: G \rightarrow G / N$ is a $\mathbb{L} \mathbf{H}$-morphism. Indeed, 1 is the unique idempotent of $G / N$ and $\pi^{-1}(1)=N$. Since $N$ is a group, its unique idempotent is 1 and the local monoid $1 N 1$ is equal to $N$. Thus $N$ belongs to $\mathbb{L} \mathbf{H}$, which proves the claim. It follows that $G$ belongs to $\mathbb{L} \mathbf{H} \mathbb{M} \mathbf{W}$ and thus $\mathbf{H} * \mathbf{W} \subseteq \mathbb{L} \mathbf{H} \mathbb{M} \mathbf{W}$.

To establish the opposite inclusion, consider a monoid $M$ in $\mathbb{L} \mathbf{H}(\mathbb{M}) \mathbf{W}$. By definition, $M$ is a quotient of a monoid $R$ for which there exists a surjective $\mathbb{L} \mathbf{H}$-morphism $\pi$ from $R$ onto a group $G$ of $\mathbf{W}$. It suffices now to prove that $R$ is a group of $\mathbf{H} * \mathbf{W}$. Let $K=\pi^{-1}(1)$. If $e$ is an idempotent of $R$, then $\pi(e)$ is idempotent and hence is equal to 1 . Therefore $K$ contains all the idempotents of $R$. It also belongs to $\mathbb{L} \mathbf{H}$ since $\pi$ is a $\mathbb{L} \mathbf{H}$-morphism. In particular, $1 K 1 \in \mathbf{H}$ and thus $K$ is a group of $\mathbf{H}$. Consequently $K$, and thus $R$, contain only one idempotent. Since a monoid with only one idempotent is a group, $R$ is a group and $\pi: R \rightarrow G$ is a group morphism with kernel $K$ in $\mathbf{H}$. Thus $R$ belongs to $\mathbf{H} * \mathbf{W}$ as required.

Our second example relates the formation of all monoids with zero to the formation $\mathbb{I L} \mathbf{G}_{p}$ of all monoids whose minimal ideal is a $p$-group.

Proposition 3.15 The formula $\mathbb{L} \mathbf{G}_{p} \mathbb{M} \mathbf{Z}=\mathbb{I L} \mathbf{G}_{p}$ holds.

Proof. Let $M$ be a monoid of $\mathbb{I L} \mathbf{G}_{p}$ : its minimal ideal $I$ belongs to $\mathbb{L} \mathbf{G}_{p}$ and $M / I$ is a monoid with zero. Let $\pi: M \rightarrow M / I$ be the quotient morphism. Then $\pi^{-1}(0)=I$ and if $s \neq 0, \pi^{-1}(s)=\{s\}$. Thus $\pi$ is a $\mathbb{L} \mathbf{G}_{p^{-}}$-morphism and $M \in \mathbb{L} \mathbf{G}_{p}(\mathbb{M}) \mathbf{Z}$.

Let now $M$ be a monoid of $\mathbb{L} \mathbf{G}_{p} \mathbb{M} \mathbf{Z}$. By Proposition 3.12, there is a surjective $\mathbb{L} \mathbf{G}_{p}$-morphism $\pi$ from $M$ onto a monoid with zero $N$. Since the minimal ideal of $M$ is a subsemigroup of $\pi^{-1}(0)$, it belongs to $\mathbb{L} \mathbf{G}_{p}$. Therefore $M \in \mathbb{I L} \mathbf{G}_{p}$. 


\section{The Formation Theorem}

\subsection{Regular languages}

In this first three paragraphs of this subsection, we make no assumption on the finiteness of the monoids. We will return to finite monoids in the fourth paragraph, for the definition of regular languages.

Recall that a monoid morphism $\varphi: A^{*} \rightarrow M$ recognises a language $L$ of $A^{*}$ if there is a subset $P$ of $M$ such that $L=\varphi^{-1}(P)$. It is equivalent to say that $L$ is saturated by $\varphi$, that is, $L=\varphi^{-1}(\varphi(L))$. If $\varphi$ is surjective, we say that $\varphi$ fully recognises $L$. By extension, one says that a language is [fully] recognised by a monoid $M$ if there exists a morphism from $A^{*}$ into $M$ which [fully] recognises $L$.

Let $L$ be a language and let $x$ and $y$ be words. The quotient $x^{-1} L y^{-1}$ of $L$ by $x$ and $y$ is defined by the formula

$$
x^{-1} L y^{-1}=\left\{u \in A^{*} \mid x u y \in L\right\}
$$

Note that if a morphism fully recognises a language $L$, then it also fully recognises its quotients.

The syntactic monoid of a language $L$ of $A^{*}$ is the quotient of $A^{*}$ by the syntactic congruence of $L$, defined on $A^{*}$ as follows: $u \sim_{L} v$ if and only if, for every $x, y \in A^{*}$,

$$
x v y \in L \Longleftrightarrow x u y \in L
$$

The natural morphism $\eta: A^{*} \rightarrow A^{*} / \sim_{L}$ is the syntactic morphism of $L$. Note that $\eta$ fully recognises $L$.

A language is regular (or recognisable) if it is recognised by some finite monoid or equivalently, if its syntactic monoid is a finite monoid. A regular language is a group language if it is recognised by some finite group or equivalently, if its syntactic monoid is a finite group.

A class of regular languages $\mathcal{C}$ associates with each finite alphabet $A$ a set $\mathcal{C}\left(A^{*}\right)$ of regular languages of $A^{*}$. It is closed under quotients if for each language $L \in \mathcal{C}\left(A^{*}\right)$ and for each pair of words $(x, y)$ of $A^{*}$, the language $x^{-1} L y^{-1}$ belongs to $\mathcal{C}$.

\subsection{Formations of languages}

The following definition was first given in [3]. A formation of languages is a class of regular languages $\mathcal{F}$ satisfying the following conditions:

$\left(\mathrm{F}_{1}\right)$ for each alphabet $A, \mathcal{F}\left(A^{*}\right)$ is closed under Boolean operations and quotients,

$\left(\mathrm{F}_{2}\right)$ if $L$ is a language of $\mathcal{F}\left(B^{*}\right)$ and $\eta: B^{*} \rightarrow M$ denotes its syntactic morphism, then for each monoid morphism $\alpha: A^{*} \rightarrow B^{*}$ such that $\eta \circ \alpha$ is surjective, the language $\alpha^{-1}(L)$ belongs to $\mathcal{F}\left(A^{*}\right)$. 
Observe that a formation of languages is closed under inverse of surjective morphisms, but this condition is not equivalent to $\left(\mathrm{F}_{2}\right)$. However, one could also use another equivalent condition:

$\left(\mathrm{F}_{2}^{\prime}\right)$ if $L$ is a language of $\mathcal{F}\left(B^{*}\right)$ and $\varphi: B^{*} \rightarrow M$ is a morphism fully recognising $L$, then for each monoid morphism $\alpha: A^{*} \rightarrow B^{*}$ such that $\varphi \circ \alpha$ is surjective, the language $\alpha^{-1}(L)$ belongs to $\mathcal{F}\left(A^{*}\right)$.

Let us also give a third equivalent definition. A class of regular languages $\mathcal{F}$ is a formation of languages if and only if it satisfies conditions $\left(\mathrm{F}_{1}\right)$ and $\left(\mathrm{F}_{3}\right)$ :

$\left(\mathrm{F}_{3}\right)$ if $L$ is a language of $\mathcal{F}\left(B^{*}\right)$ and $K$ is a language of $A^{*}$ whose syntactic monoid is a quotient of the syntactic monoid of $L$, then $K$ belongs to $\mathcal{F}\left(A^{*}\right)$.

To each formation of monoids $\mathbf{F}$, let us associate the class of languages $\mathcal{F}(\mathbf{F})$ defined as follows: for each alphabet $A, \mathcal{F}(\mathbf{F})\left(A^{*}\right)$ is the set of languages of $A^{*}$ fully recognised by some monoid of $\mathbf{F}$, or, equivalently, whose syntactic monoid belongs to $\mathbf{F}$.

Given a formation of languages $\mathcal{F}$, let us denote by $\mathbf{F}(\mathcal{F})$ the formation of monoids generated by the syntactic monoids of the languages of $\mathcal{F}$. The following statement is the main result of [3].

Theorem 4.1 (Formation Theorem) The correspondences $\mathbf{F} \rightarrow \mathcal{F}(\mathbf{F})$ and $\mathcal{F} \rightarrow \mathbf{F}(\mathcal{F})$ are two mutually inverse, order preserving, bijections between formations of monoids and formations of languages.

As an example, let us describe the formation of languages corresponding to $\mathbf{Z}$, the formation of monoids having a zero. Recall that a language $L$ of $A^{*}$ is nondense if there exists a word $u \in A^{*}$ which cannot be completed into a word of $L$, that is, such that $L \cap A^{*} u A^{*}=\emptyset$. A language is co-nondense if its complement is nondense.

Proposition 4.2 The formation of languages corresponding to $\mathbf{Z}$ consists of the regular nondense or co-nondense languages.

Proof. Let $L$ be a regular nondense language and let $\eta: A^{*} \rightarrow M$ be the syntactic monoid of $L$. Let $u$ be a word of $A^{*}$ such that $L \cap A^{*} u A^{*}=\emptyset$. Then for all $x \in A^{*}, x u \sim_{L} u \sim_{L} u x$ and hence $\eta(u)$ is a zero in $M$. If $L$ is co-nondense, then its syntactic monoid is equal to the syntactic monoid, which has a zero.

Let $M$ be a monoid with zero and let $L$ be a language recognised by a surjective morphism $\varphi: A^{*} \rightarrow M$. Also let $u$ be a word such that $\varphi(u)=0$. If $0 \notin \varphi(L)$, then $u$ cannot be completed into a word of $L$ and thus $L$ is nondense. If $0 \in \varphi(L)$, then $0 \notin \varphi\left(A^{*}-L\right)$ since $L=\varphi^{-1}(\varphi(L))$. It follows that $A^{*}-L$ is nondense and thus $L$ is co-nondense. 


\subsection{Languages of saturated formations of groups}

Let $\mathbf{F}$ be a saturated formation of groups and let $F$ be its canonical local definition. By virtue of [3, Theorem 4.2], each formation of groups $F(p)$ is associated with a formation of languages $\mathcal{F}_{p}$. Lemma 2.1$]$ allows one to describe the formation of languages $\mathcal{F}$ associated with $\mathbf{F}$.

Corollary 4.3 The formation of languages $\mathcal{F}$ is the join of the formations of languages $\mathcal{F}_{p}$, for all primes $p$.

Corollary 4.3 shows that computing $\mathcal{F}$ amounts to computing $\mathcal{F}_{p}$ for all primes $p$. We know that $F(p)=\mathbf{G}_{p} *(f(p) \cap \mathbf{F})$, for all primes $p$, where $f$ is an arbitrary local definition of $\mathbf{F}$. Further, Proposition 3.14 shows that $\mathbf{G}_{p} * \mathbf{F}=\mathbb{L} \mathbf{G}_{p} \mathbb{(} \mathbb{M}$. It now remains to describe the formation of languages corresponding to $\mathbb{L} \mathbf{G}_{p} \mathbb{M} \mathbf{F}$, given the formation of languages corresponding to $\mathbf{F}$. The solution to this problem relies on an operation on languages first introduced in [24], the modular product, which is the topic of the next section.

\section{$5 \quad$ Modular product of languages}

Let $p$ be a prime number. Let $L_{0}, \ldots, L_{k}$ be languages of $A^{*}$, let $a_{1}, \ldots, a_{k}$ be letters of $A$. Let also $r$ be an integer such that $0 \leqslant r<p$. We define the modular product of the languages $L_{0}, \ldots, L_{k}$ with respect to $r$ and $p$, denoted $\left(L_{0} a_{1} L_{1} \cdots a_{k} L_{k}\right)_{r, p}$, as the set of all words $u$ in $A^{*}$ such that the number of factorizations of $u$ in the form $u=u_{0} a_{1} u_{1} \cdots a_{k} u_{k}$, with $u_{i} \in L_{i}$ for $0 \leqslant i \leqslant k$, is congruent to $r$ modulo $p$. A language is a $p$-modular product of the languages $L_{0}, \ldots, L_{k}$ if it is of the form $\left(L_{0} a_{1} L_{1} \cdots a_{k} L_{k}\right)_{r, p}$ for some $r$.

\subsection{Péladeau's results}

In this section, we briefly survey the results of Péladeau [10]. They were originally stated for varieties of languages but can be readily extended to a Boolean algebra of regular languages closed under quotient.

Let $\mathbb{F}_{p}$ be the $p$-element field and let $F\left(A^{*}, \mathbb{F}_{p}\right)$ be the ring of all functions from $A^{*}$ to $\mathbb{F}_{p}$. We consider in particular the functions $\left[L_{0}, a_{1}, L_{1}, \cdots a_{k}, L_{k}\right]_{p}$ : $A^{*} \rightarrow \mathbb{F}_{p}$ which map a word $u$ to the residue modulo $p$ of the number of distinct factorizations of $u$ in the form $u=u_{0} a_{1} u_{1} \cdots a_{k} u_{k}$, with $u_{i} \in L_{i}$ for $0 \leqslant i \leqslant k$.

Let $\mathcal{B}$ be a Boolean algebra of regular languages of $A^{*}$. We denote by $\operatorname{Pol}_{p}(\mathcal{B})$ the set of all languages which can be written as a finite union of $p$ modular products of languages of $\mathcal{B}$ and by $\operatorname{Pol}_{p}\left(\mathcal{B}, \mathbb{F}_{p}\right)$ the vector space (over $\mathbb{F}_{p}$ ) of all linear combinations of functions of the form $\left[L_{0}, a_{1}, L_{1}, \cdots a_{k}, L_{k}\right]_{p}$, with $L_{0}, \ldots, L_{k}$ in $\mathcal{B}$. 
Note that since $L=(L)_{1, p}, \operatorname{Pol}_{p}(\mathcal{B})$ always contains $\mathcal{B}$. Péladeau's results can be summarized as follows.

Theorem 5.1 (Péladeau) Let $\mathcal{B}$ be a Boolean algebra of languages of $A^{*}$ closed under quotients. Then the following properties hold:

(1) $\operatorname{Pol}_{p}(\mathcal{B})$ is a Boolean algebra,

(2) $\operatorname{Pol}_{p}\left(\mathcal{B}, \mathbb{F}_{p}\right)$ is a subring of $F\left(A^{*}, \mathbb{F}_{p}\right)$,

(3) A language $L$ belongs to $\operatorname{Pol}_{p}(\mathcal{B})$ if and only if there is a function $f \in \operatorname{Pol}_{p}\left(\mathcal{B}, \mathbb{F}_{p}\right)$ such that $L=f^{-1}(1)$,

(4) $\operatorname{Pol}_{p}\left(\operatorname{Pol}_{p}(\mathcal{B})\right)=\operatorname{Pol}_{p}(\mathcal{B})$.

\subsection{Schützenberger products and modular product}

An algebraic tool adapted to the study of the $p$-modular product is the Schützenberger product over $\mathbb{F}_{p}$ of a family of monoids.

Let $M_{0}, \ldots, M_{k}$ be monoids. Denote by $K=\mathbb{F}_{p}\left[M_{0} \times \cdots \times M_{k}\right]$ the monoid algebra of $M_{0} \times \cdots \times M_{k}$ over $\mathbb{F}_{p}$ and by $M_{k+1}(K)$ the multiplicative monoid of square matrices of size $k+1$ with entries in $K$. The Schützenberger product over $\mathbb{F}_{p}$ of the monoids $M_{0}, \ldots, M_{k}$, denoted by $\mathbb{F}_{p} \diamond\left(M_{0}, \ldots, M_{k}\right)$, is the submonoid of $M_{k+1}(K)$ made up of matrices $m=\left(m_{i, j}\right)$ such that

(1) $m_{i, j}=0$, for $i>j$,

(2) $m_{i, i}=\left(1, \ldots, 1, m_{i}, 1, \ldots, 1\right)$ for some $m_{i} \in M_{i}$,

(3) $m_{i, j} \in \mathbb{F}_{p}\left[1 \times \cdots \times 1 \times M_{i} \times \cdots \times M_{j} \times 1 \times \cdots \times 1\right]$, for $i<j$.

It turns out that $\mathbb{F}_{p} \diamond\left(M_{0}, \ldots, M_{k}\right)$ recognises the $p$-modular products of languages recognised by $M_{0}, \ldots, M_{k}$. More specifically, let, for $0 \leqslant i \leqslant k$, $L_{i}$ be a language of $A^{*}$ and let $\eta_{i}: A^{*} \rightarrow M_{i}$ be its syntactic morphism. Let $p$ be a prime, let $a_{1}, \ldots, a_{k}$ be letters of $A$ and let $L=\left(L_{0} a_{1} L_{1} \cdots a_{k} L_{k}\right)_{r, p}$ where $0 \leqslant r<p$. Let $\mu: A^{*} \rightarrow \mathbb{F}_{p} \diamond\left(M_{0}, \ldots, M_{k}\right)$ be the morphism defined for each letter $a \in A$ by

$$
\begin{aligned}
\mu_{i, i}(a) & =\left(1, \ldots, 1, \eta_{i}(a), 1, \ldots, 1\right) & & \text { for } 0 \leqslant i \leqslant k \\
\mu_{i, i+1}(a) & =(1, \ldots, 1) \quad \text { if } a=a_{i+1} & & \text { for } 0 \leqslant i \leqslant k-1 \\
\mu_{i, j}(a) & =0 \quad \text { otherwise. } & &
\end{aligned}
$$

It follows immediately from the definition that, for each $u \in A^{*}$, and for $0 \leqslant i \leqslant k, \mu_{i, i}(u)$ is equal to $\left(1, \ldots, 1, \eta_{i}(u), 1, \ldots, 1\right)$ and therefore can be identified with the element $\eta_{i}(u)$ of $M_{i}$. We can now state [12, 14, 31]:

Proposition 5.2 The language $\left(L_{0} a_{1} L_{1} \cdots a_{k} L_{k}\right)_{r, p}$ is recognised by the morphism $\mu: A^{*} \rightarrow \mathbb{F}_{p} \diamond\left(M_{0}, \ldots, M_{k}\right)$.

We shall use an algebraic property due to Weil [32, Corollary 3.6]. Let $\pi: \mathbb{F}_{p} \diamond\left(M_{0}, \ldots, M_{k}\right) \rightarrow M_{0} \times \cdots \times M_{k}$ be the morphism which maps each matrix onto its diagonal. 
Proposition 5.3 The morphism $\pi$ is a $\mathbb{L} \mathbf{G}_{p}$-morphism.

For $k=2$, a more precise result holds, as a consequence of [1, Lemma 5.4].

Proposition 5.4 The morphism $\pi: \mathbb{F}_{p} \gg\left(M_{0}, M_{1}\right) \rightarrow M_{0} \times M_{1}$ is a $\ell \mathbf{A b}(p)$ morphism.

One can actually use Propositions 5.3 and 5.4 to give another proof of Propositions 5.6 and 5.5, respectively.

\subsection{Relational morphisms and modular product}

An important question regarding the modular product is to understand the algebraic relations between the syntactic monoids of the languages $L_{0}, \ldots$, $L_{k}$ on the one hand, and the syntactic monoid of $\left(L_{0} a_{1} L_{1} \cdots a_{k} L_{k}\right)_{r, p}$ on the other hand. We first treat the case $k=1$.

Let $\eta_{0}: A^{*} \rightarrow M\left(L_{0}\right)$ and $\eta_{1}: A^{*} \rightarrow M\left(L_{1}\right)$ be the syntactic morphisms of $L_{0}$ and $L_{1}$ respectively and let $\eta: A^{*} \rightarrow M\left(L_{0}\right) \times M\left(L_{1}\right)$ be the morphism defined by $\eta(u)=\left(\eta_{0}(u), \eta_{1}(u)\right)$. Let $a$ be a letter of $A$, let $L=\left(L_{0} a L_{1}\right)_{r, p}$ and let $\gamma: A^{*} \rightarrow M(L)$ be the syntactic morphism of $L$. Consider the relational morphism $\tau=\eta \circ \gamma^{-1}: M(L) \rightarrow M\left(L_{0}\right) \times M\left(L_{1}\right)$.

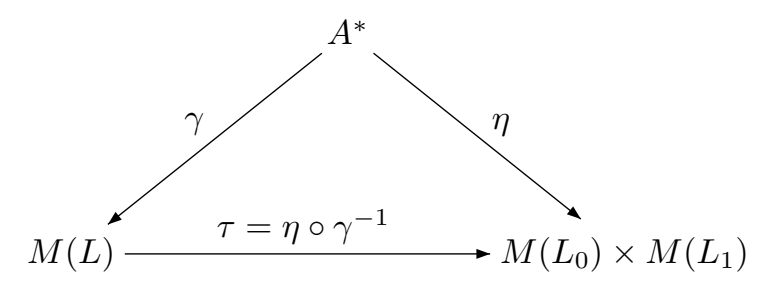

The following result is inspired by the results of Straubing [25].

Proposition 5.5 The relational morphism $\tau: M(L) \rightarrow M\left(L_{0}\right) \times M\left(L_{1}\right)$ is a relational $\ell \mathbf{A b}(p)$-morphism.

Proof. We must show that for each $x_{0}, x_{1}, u, v \in A^{*}$ such that

$$
x_{0} u \sim_{L_{0}} x_{0} v \sim_{L_{0}} x_{0} \text { and } u x_{1} \sim_{L_{1}} v x_{1} \sim_{L_{1}} x_{1},
$$

one has $x_{0} u v x_{1} \sim_{L} x_{0} v u x_{1}$ and $x_{0} u^{p} x_{1} \sim_{L} x_{0} x_{1}$. Given $s, t \in A^{*}$, we set $f=s x_{0} u v x_{1} t$ and $g=s x_{0} v u x_{1} t$. Consider a factorization of $f$ of the form $z_{0} a z_{1}$ with $z_{0} \in L_{0}$ and $z_{1} \in L_{1}$. We call such a factorization fit. Depending of the position of $a$ in this factorization, we obtain a factorization of $g$ as follows:

(1) if $a$ occurs on the left of $u v$, that is, if $z_{1}=z_{1}^{\prime} u v x_{1} t$ for some $z_{1}^{\prime} \in A^{*}$, then we take $g=\left(z_{0}\right) a\left(z_{1}^{\prime} v u x_{1} t\right)$,

(2) if $a$ occurs on the right of $u v$, that is, if $z_{0}=s x_{0} u v z_{0}^{\prime}$ for some $z_{0}^{\prime} \in A^{*}$, then we take $g=\left(s x_{0} v u z_{0}^{\prime}\right) a\left(z_{1}\right)$, 
(3) if $a$ occurs inside $u$, that is, if $z_{0}=s x_{0} u^{\prime}$ and $z_{1}=u^{\prime \prime} v x_{1} t$, with $u=u^{\prime} a u^{\prime \prime}$, then we take $g=\left(s x_{0} v u^{\prime}\right) a\left(u^{\prime \prime} x_{1} t\right)$,

(4) if $a$ occurs inside $v$, that is, if $z_{0}=s x_{1} u v^{\prime}$ and $z_{1}=v^{\prime \prime} x_{1} t$, with $v=v^{\prime} a v^{\prime \prime}$, then we take $g=\left(s x_{1} v^{\prime}\right) a\left(v^{\prime \prime} u x_{1} t\right)$.

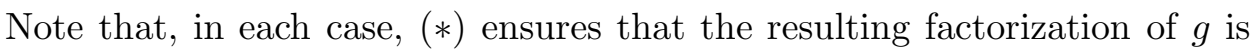
fit. This correspondence actually defines a bijection between the fit factorizations of $f$ and $g$. It follows that $f$ and $g$ have exactly the same number of fit factorizations and hence $f \in L$ if and only if $g \in L$. This proves that $x_{0} u v x_{1} \sim_{L} x_{0} v u x_{1}$.

We now prove that $x_{0} u^{p} x_{1} \sim_{L} x_{0} x_{1}$. Given $s, t \in A^{*}$, we set $f=$ $s x_{0} u^{p} x_{1} t$ and $g=s x_{0} x_{1} t$. We claim that the number of fit factorizations of $f$ is congruent modulo $p$ to the the number of fit factorizations of $g$. We consider again the position of $a$ in a fit factorization $z_{0} a z_{1}$ of $f$. If $a$ occurs on the left of $u^{p}$, that is, if $z_{1}=z_{1}^{\prime} u^{p} x_{1} t$ for some $z_{1}^{\prime} \in A^{*}$, then $\left(z_{0}\right) a\left(z_{1}^{\prime} x_{1} t\right)$ is a fit factorization of $g$. Similarly, if $a$ occurs on the right of $u^{p}$, that is, if $z_{0}=s x_{0} u^{p} z_{0}^{\prime}$ for some $z_{0}^{\prime} \in A^{*}$, then $\left(s x_{0} z_{0}^{\prime}\right) a\left(z_{1}\right)$ is a fit factorization of $g$. It follows that the number of fit factorizations of $f$ in which $a$ does not occur in $u^{p}$ is equal to the number of fit factorizations of $g$.

Suppose now that $a$ occurs in one of the factors $u$, that is, $z_{0}=s x_{0} u^{i} u^{\prime}$, $z_{1}=u^{\prime \prime} u^{j} x_{1} t$ with $u^{\prime} a u^{\prime \prime}=u$ and $i+j+1=p$. Since $p$ is a prime, one has $p \geqslant 2$ and thus $i+j \geqslant 1$. Therefore each fit factorization of $f$ of this type gives rise to $p$ fit factorizations of $f$, given by $\left(s x_{0} u^{k} u^{\prime}\right) a\left(u^{\prime \prime} u^{\ell} x_{1} t\right)$ with $k+\ell=p-1$. It follows that the number of fit factorizations of $f$ in which $a$ occurs inside $u^{p}$ is a multiple of $p$. This proves the claim and shows that $f \in L$ if and only if $g \in L$. It follows that $x_{0} u^{p} x_{1} \sim_{L} x_{0} x_{1}$ as required.

In the general case where $L=\left(L_{0} a_{1} L_{1} \cdots a_{k} L_{k}\right)_{r, p}$, a slightly weaker result holds. Let, for $0 \leqslant i \leqslant k, \eta_{i}: A^{*} \rightarrow M\left(L_{i}\right)$ be the syntactic morphism of $L_{i}$ and let $N=M\left(L_{0}\right) \times \cdots \times M\left(L_{k}\right)$. Let $\eta: A^{*} \rightarrow N$ be the morphism defined by $\eta(u)=\left(\eta_{0}(u), \cdots, \eta_{k}(u)\right)$ and let $\tau: M(L) \rightarrow N$ be the relational morphism defined by $\tau=\eta \circ \gamma^{-1}$.

Proposition 5.6 The relational morphism $\tau: M(L) \rightarrow M\left(L_{0}\right) \times \cdots \times$ $M\left(L_{k}\right)$ is a relational $\mathbb{L} \mathbf{G}_{p}$-morphism.

Proof. Let $\mu: A^{*} \rightarrow \mathbb{F}_{p} \diamond\left(M_{0}, \ldots, M_{k}\right)$ and let $T=\mu\left(A^{*}\right)$. Recall that $\pi: \mathbb{F}_{p} \diamond\left(M_{0}, \ldots, M_{k}\right) \rightarrow N$ denotes the morphism which maps each matrix onto its diagonal. Since $\pi \circ \mu=\eta$, one has $\pi(T)=N$. By Proposition [5.2, the monoid $T$ fully recognises $L$ and thus there is a surjective morphism $\theta: T \rightarrow M(L)$ such that $\gamma=\theta \circ \mu$. 


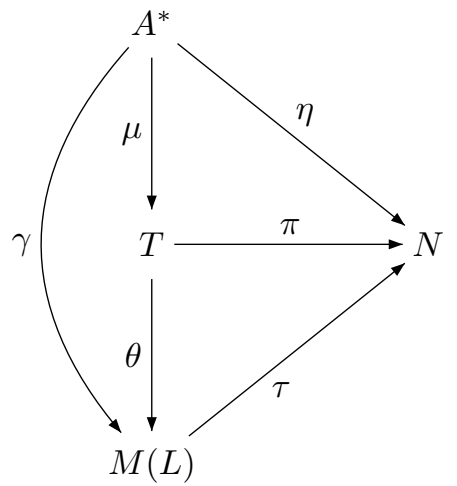

We also have $\tau^{-1}=\gamma \circ \eta^{-1}=\theta \circ \mu \circ \eta^{-1}=\theta \circ \pi^{-1}$. Let $e$ be an idempotent of $N$ and let $S=\tau^{-1}(e)=\theta\left(\pi^{-1}(e)\right)$. Since $\pi$ is a $\mathbb{L} \mathbf{G}_{p}$-morphism by Proposition 5.3, $\pi^{-1}(e)$ belongs to $\mathbb{L} \mathbf{G}_{p}$. Since $\mathbb{L} \mathbf{G}_{p}$ is a variety, $S$ also belongs to $\mathbb{L} \mathbf{G}_{p}$ and thus $\tau$ is a relational $\mathbb{L} \mathbf{G}_{p}$-morphism.

Propositions 5.5 and 5.6 give immediately the following corollaries. Let $\mathbf{F}$ be a formation of monoids and let $\mathcal{F}$ be the corresponding formation of languages.

Corollary 5.7 Let $L_{0}$ and $L_{1}$ be languages of $\mathcal{F}\left(A^{*}\right)$. Then the syntactic monoid of the modular product $\left(L_{0} a_{1} L_{1}\right)_{r, p}$ belongs to $\ell \mathbf{A b}(p) \mathbb{}(\mathbb{N} \mathbf{F}$.

Corollary 5.8 Let $L_{0}, \ldots, L_{k}$ be languages of $\mathcal{F}\left(A^{*}\right)$. Then the syntactic monoid of the modular product $\left(L_{0} a_{1} L_{1} \cdots a_{k} L_{k}\right)_{r, p}$ belongs to $\mathbb{L} \mathbf{G}_{p} \mathbb{M} \mathbf{F}$.

The next propositions can be viewed as a partial converse to Propositions 5.5 and 5.6, respectively. This result and its proof are inspired by the corresponding result for the unambiguous product [15, Proposition 2.2].

Theorem 5.9 Let $\pi: M \rightarrow N$ be a surjective $\ell \mathbf{A b}(p)$-morphism. Then every language fully recognised by $M$ is a finite union of finite intersections of languages fully recognised by $N$ and of modular products $\left(L_{0} a L_{1}\right)_{r, p}$, where $L_{0}$ and $L_{1}$ are fully recognised by $N$.

Proof. Let $\mathcal{B}$ be the set of languages that are finite union of finite intersections of languages fully recognised by $N$ and of modular products $\left(L_{0} a L_{1}\right)_{r, p}$, where $L_{0}$ and $L_{1}$ are fully recognised by $N$. We claim that $\mathcal{B}$ is actually a Boolean algebra. It suffices to prove that the complements of the generators of $\mathcal{B}$ are also in $\mathcal{B}$. Indeed, the complement of a language fully recognised by $N$ is also fully recognised by $N$. Further,

$$
\left(L_{0} a L_{1}\right)_{r, p}^{c}=\bigcup_{\substack{0 \leqslant s<p \\ s \neq r}}\left(L_{0} a L_{1}\right)_{s, p}
$$


Let $\equiv$ be the relation on $A^{*}$ defined by $u \equiv v$ if and only if, for all languages $L, L_{0}$ and $L_{1}$ fully recognised by $N$ and for all $r$ such that $0 \leqslant r<p$, one has

$$
\begin{aligned}
u \in L & \Longleftrightarrow v \in L \\
u \in\left(L_{0} a L_{1}\right)_{r, p} & \Longleftrightarrow v \in\left(L_{0} a L_{1}\right)_{r, p}
\end{aligned}
$$

Lemma 5.10 The relation $\equiv$ is a congruence of finite index on $A^{*}$.

Proof. We first prove that $\equiv$ is a congruence. It suffices to show that if $c$ is a letter, $u \equiv v$ implies $c u \equiv c v$ and $u c \equiv v c$. By symmetry, it suffices to prove the first property. Let $L$ be a language fully recognised by $N$. If $c u \in L$, then $u \in c^{-1} L$, and since $c^{-1} L$ is fully recognised by $N$, this is equivalent to $v \in c^{-1} L$ and finally to $c v \in L$. Thus $c u \in L$ if and only if $c v \in L$.

Assume now that $c u \in\left(L_{0} a L_{1}\right)_{r, p}$. Suppose first that $u$ meets the following condition:

(1) every factorization of $c u$ in $L_{0} a L_{1}$ is of the form $c x_{0} a x_{1}$ with $c x_{0} \in L_{0}$ and $x_{1} \in L_{1}$.

Then each factorization of $c u$ in $L_{0} a L_{1}$ yields a factorization of $u$ in $\left(c^{-1} L_{0}\right) a L_{1}$. It follows that $u \in\left(\left(c^{-1} L_{0}\right) a L_{1}\right)_{r, p}$, which is equivalent to $v \in\left(\left(c^{-1} L_{0}\right) a L_{1}\right)_{r, p}$ and finally to $c v \in\left(L_{0} a L_{1}\right)_{r, p}$. The only case where Condition (1) is not satisfied is when $c=a$, the empty word belongs to $L_{0}$ and $u$ belongs to $L_{1}$. In this case, $1 a u$ is another factorization of $c u$ in $\left(L_{0} a L_{1}\right)_{r, p}$. It follows that $u$ belongs to $\left(\left(c^{-1} L_{0}\right) a L_{1}\right)_{r-1, p}$. But then again, this is equivalent to $v \in\left(\left(c^{-1} L_{0}\right) a L_{1}\right)_{r-1, p}$ and then to $c v \in\left(L_{0} a L_{1}\right)_{r, p}$. Thus $c u \equiv c v$ in all cases.

Finally, since there are $|N|^{|A|}$ functions from the finite alphabet $A$ to the finite monoid $N$, there are $|N|^{|A|}$ morphisms from $A^{*}$ to $N$. Therefore, there are finitely many languages fully recognised by $N$ and finitely many languages of the form $\left(L_{0} a L_{1}\right)_{r, p}$ with $L_{0}$ and $L_{1}$ fully recognised by $N$. It follows that the equivalence $\equiv$ has finite index.

Let $\varphi: A^{*} \rightarrow M$ be a surjective morphism.

Lemma 5.11 If $u \equiv v$ implies $\varphi(u)=\varphi(v)$, then every language recognised by $\varphi$ is a Boolean combination of languages fully recognised by $N$ and of modular products $\left(L_{0} a_{1} L_{1}\right)_{r, p}$, where $L_{0}$ and $L_{1}$ are fully recognised by $N$.

Proof. Suppose that $u \equiv v$ implies $\varphi(u)=\varphi(v)$. Then every language recognised by $\varphi$ is a finite union of $\equiv$-classes. By construction of $\equiv$, an 三-class is a Boolean combination of languages fully recognised by $N$ and of modular products $\left(L_{0} a_{1} L_{1}\right)_{r, p}$, where $L_{0}$ and $L_{1}$ are fully recognised by $N$. 
It remains to prove that $u \equiv v$ implies $\varphi(u)=\varphi(v)$. We start by proving a weaker property. Let $\mu=\pi \circ \varphi$.

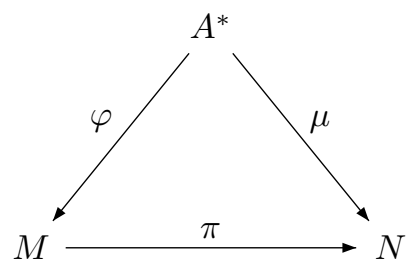

Lemma 5.12 If $u \equiv v$ then $\mu(u)=\mu(v)$.

Proof. Let $n=\mu(u)$ and let $L=\mu^{-1}(n)$. By construction, $L$ is fully recognised by $N$ and $u \in L$. If $u \equiv v$, then $v \in L$ and thus $\mu(v)=n$. Therefore $\mu(u)=\mu(v)$.

Observe now that $A$ acts on $N$ on the left and on the right by setting $n a=n \mu(a)$ and $a n=\mu(a) n$. Let $C$ be the category whose objects are the pairs $\left(n_{0}, n_{1}\right)$ with $\left(n_{0}, n_{1}\right) \in N \times N$ and whose arrows are of the form

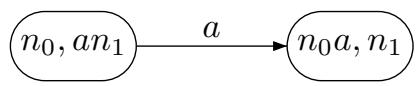

Each word $u=b_{1} \cdots b_{n}$, where $b_{1}, \ldots, b_{n} \in A$, defines a path $p(u)$ in $C$ :

$$
p(u)=(\mu(1), \mu(u)) \stackrel{b_{1}}{\longrightarrow}\left(\mu\left(b_{1}\right), \mu\left(b_{2} \cdots b_{n}\right)\right) \stackrel{b_{2}}{\longrightarrow} \cdots \stackrel{b_{n}}{\longrightarrow}(\mu(u), \mu(1))
$$

If $p$ is a path in $C$ and $e$ is an arrow, we denote by $|p|_{e}$ the number of occurrences of $e$ in $p$.

Lemma 5.13 If $u \equiv v$, then the paths $p(u)$ and $p(v)$ are coterminal and satisfy $|p(u)|_{e} \equiv|p(v)|_{e} \bmod p$ for each arrow e of $C$.

Proof. If $u \equiv v$, then $\mu(u)=\mu(v)$ by Lemma 5.12 and thus the paths $p(u)$ and $p(v)$ are coterminal. Let $e=\left(\left(n_{0}, a n_{1}\right), a,\left(n_{0} a, n_{1}\right)\right)$ be an arrow of $C$ and let $L_{0}=\mu^{-1}\left(n_{0}\right)$ and $L_{1}=\mu^{-1}\left(n_{1}\right)$. There is a natural bijection between the occurrences of $e$ in $p(u)$ and the factorisations of $u$ in $L_{0} a L_{1}$. But since $u \equiv v$, one has for $0 \leqslant r<p, u \in\left(L_{0} a L_{1}\right)_{r, p}$ if and only if $v \in\left(L_{0} a L_{1}\right)_{r, p}$. Therefore $|p(u)|_{e} \equiv|p(v)|_{e} \bmod p$.

We now need a graph-theoretic result inspired by an analogous result of Simon [22]. We mix freely the vocabulary of graph theory and category theory in the next statement and its proof.

Lemma 5.14 Let $C$ be a finite graph and let $C^{*}$ be the free category on $C$. Let $\sim$ be a congruence of category on $C^{*}$ such that, for all loops $s$ and $t$ around the same vertex $u, s^{p} \sim 1_{u}$ and st $\sim$ ts. Let $x, y$ be two coterminal paths such that, for each $e$ in $C,|x|_{e} \equiv|y|_{e} \bmod p$. Then $x$ and $y$ are $\sim$-equivalent. 
Lemma 5.14 actually follows from the results of Straubing [26] and Thérien [29], but we give a self-contained proof for the convenience of the reader. We use freely the graph-theoretic notions of vertices and edges rather than objects and arrows.

Proof. Suppose that $x$ and $y$ are coterminal paths from $u$ to $v$ and let $c(x)$ $[c(y)]$ be the set of edges of $x[y]$. Let us prove the lemma by induction on $n=|c(x)|+|c(y)|$, the case $n=0$ being trivial. Let $G$ be the subgraph of $C$ consisting of the edges of $c(x) \cup c(y)$. We need to consider separately two cases, depending on whether $G$ is strongly connected or not. Let $\{0, \ldots, n\}$ be the set of vertices of $G$.

If $G$ is strongly connected, let us fix for each vertex $v$ of $G$ a path $r_{v, 0}$ from $v$ to 0 and a path $r_{0, v}$ from 0 to $v$. Let $\operatorname{Loc}(0)$ be the local monoid of $C^{*}$ at 0 and let $\varphi: C \rightarrow \operatorname{Loc}(0)$ be the map defined as follows: if $e \in C$ is an edge from $i$ to $j$, then $\varphi(e)=r_{0, i} e\left(r_{j, 0} r_{0, j}\right)^{p-1} r_{j, 0}$.

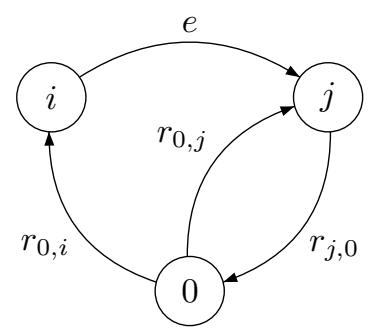

The map $\varphi$ extends uniquely to a category morphism from $C^{*}$ to $\operatorname{Loc}(0)$. Observe that if $\left(i, e_{1}, j\right)$ and $\left(j, e_{2}, k\right)$ are two consecutive edges, then

$$
\begin{aligned}
\varphi\left(e_{1} e_{2}\right) & =\left(r_{0, i} e_{1}\left(r_{j, 0} r_{0, j}\right)^{p-1} r_{j, 0}\right)\left(r_{0, j} e_{2}\left(r_{k, 0} r_{0, k}\right)^{p-1} r_{k, 0}\right) \\
& =r_{0, i} e_{1}\left(r_{j, 0} r_{0, j}\right)^{p} e_{2}\left(r_{k, 0} r_{0, k}\right)^{p-1} r_{k, 0} \sim r_{0, i} e_{1} e_{2}\left(r_{k, 0} r_{0, k}\right)^{p-1} r_{k, 0}
\end{aligned}
$$

since $r_{j, 0} r_{0, j}$ is a loop around 0 . Thus $e_{1} e_{2} \sim\left(r_{i, 0} r_{0, i}\right)^{p-1} r_{i, 0} \varphi\left(e_{1} e_{2}\right) r_{0, k}$. More generally, if $x$ is a path from $u$ to $v$, then $x \sim\left(r_{u, 0} r_{0, u}\right)^{p-1} r_{u, 0} \varphi(x) r_{0, v}$.

Let now $x$ and $y$ be two paths from $u$ to $v$ such that $|x|_{e} \equiv|y|_{e} \bmod p$ for each $e$ in $C$. It follows from the definition of the congruence $\sim$ that $\varphi(x) \sim$ $\varphi(y)$ and thus $x \sim r_{u, 0}\left(r_{0, u} r_{u, 0}\right)^{p-1} \varphi(x) r_{0, v} \sim r_{u, 0}\left(r_{0, u} r_{u, 0}\right)^{p-1} \varphi(y) r_{0, v} \sim y$.

Suppose now that $G$ is not strongly connected. Then there is an edge $a$ of $G$ from $i$ to $j$ for which there is no path from $j$ to $i$. Without loss of generality, we may assume that $a$ is an edge of $x$. Thus there is a factorisation $x=x_{0} a x_{1}$ such that $\left|x_{0}\right|_{a}=\left|x_{1}\right|_{a}=0$ and $c(x)$ is the disjoint union of $c\left(x_{0}\right)$, $\{a\}$ and $c\left(x_{1}\right)$. Since $|x|_{a} \equiv|y|_{a} \bmod p$, the vertex $a$ also occurs in $y$ and there is a factorisation $y=y_{0} a y_{1}$. Further, an edge of $y_{1}$ can not belong to $c\left(x_{0}\right) \cup c\left(y_{0}\right)$, otherwise, there would be a path from $j$ to $i$. Similarly, an edge of $y_{0}$ does not belong to $c\left(x_{1}\right) \cup c\left(y_{1}\right)$ for the same reason. It follows that for each edge $e,\left|x_{0}\right|_{e}=\left|y_{0}\right|_{e}$ and $\left|x_{1}\right|_{e}=\left|y_{1}\right|_{e}$. Therefore $x_{0} \sim y_{0}$ and $x_{1} \sim y_{1}$ by the induction hypothesis, and finally $x \sim y$. 
We now apply Lemma 5.14 to the congruence $\sim$ on $C^{*}$ defining $\operatorname{ker}(\pi)$. This congruence satisfies the condition $s^{p} \sim 1$ and $s t \sim t s$ for all loops around the same object. Consequently, if $u \equiv v$, then by Lemma 5.13, $u$ and $v$ define equal paths in $\operatorname{ker}(\pi)$ from $(1, \mu(u))$ to $(\mu(u), 1)$. In particular, $1 \varphi(u) 1=1 \varphi(v) 1$, that is, $\varphi(u)=\varphi(v)$. This concludes the proof of Proposition 5.9.

\section{Formations of languages and modular product}

The aim of this section is to describe the algebraic counterpart to the closure of a formation of languages under modular product. The next two results extend and improve the results of $[1,10,31,32$.

Theorem 6.1 Let $\mathbf{F}$ be a formation of monoids and let $\mathbf{W}=\ell \mathbf{A} \mathbf{b}(p) \mathbb{M}$. Let $\mathcal{F}$ and $\mathcal{W}$ be the formations of languages corresponding to $\mathbf{F}$ and $\mathbf{W}$, respectively. Then, for each alphabet $A, \mathcal{W}\left(A^{*}\right)$ is the Boolean algebra generated by the languages of $\mathcal{F}\left(A^{*}\right)$ and by the modular products $\left(L_{0} a_{1} L_{1}\right)_{r, p}$, where $L_{0}, L_{1}$ belong to $\mathcal{F}\left(A^{*}\right)$.

Proof. This is a direct consequence of Proposition 5.9.

Theorem 6.2 Let $\mathbf{F}$ be a formation of monoids and let $\mathbf{W}=\mathbb{L} \mathbf{G}_{p} \mathbb{M} \mathbf{F}$. Let $\mathcal{F}$ and $\mathcal{W}$ be the formations of languages corresponding to $\mathbf{F}$ and $\mathbf{W}$, respectively. Then, for each alphabet $A, \mathcal{W}\left(A^{*}\right)$ is

(1) the lattice generated by the p-modular products of members of $\mathcal{F}\left(A^{*}\right)$,

(2) the Boolean algebra generated by the p-modular products of members of $\mathcal{F}\left(A^{*}\right)$,

Further, $\mathcal{W}\left(A^{*}\right)$ is closed under $p$-modular products.

Property (2) is proved for varieties of languages in [1, Corollary 6.3] and [32, Corollary 4.5]. Property (1) is implicitly proved in [10], again only for varieties of languages.

Proof. Corollary 5.8 shows that any $p$-modular product of languages of $\mathcal{F}\left(A^{*}\right)$ belong to $\mathcal{W}\left(A^{*}\right)$. Since $\mathcal{W}$ is a formation of languages, it follows that the classes of languages $\mathcal{W}_{1}$ and $\mathcal{W}_{2}$, defined respectively by the conditions $(1)$ and $(2)$, are contained in $\mathcal{W}$. Further, the complement of a $p$-modular product is a finite union of $p$-modular products since, for $0 \leqslant r<p$, one gets

$$
\left(L_{0} a_{1} L_{1} \cdots a_{k} L_{k}\right)_{r, p}^{c}=\bigcup_{\substack{0 \leqslant s<k \\ s \neq r}}\left(L_{0} a_{1} L_{1} \cdots a_{k} L_{k}\right)_{s, p}
$$

It follows that $\mathcal{W}_{1}=\mathcal{W}_{2}$ and it suffices now to prove the inclusion $\mathcal{W} \subseteq \mathcal{W}_{2}$. 
Let $L$ be language of $\mathcal{W}\left(A^{*}\right)$. By definition, its syntactic monoid $M$ belongs to $\mathbb{L} \mathbf{G}_{p} \mathbb{M} \mathbf{F}$ and by Proposition 3.12 , there is a surjective $\mathbb{L} \mathbf{G}_{p^{-}}$ morphism $\pi: M \rightarrow N$, where $N$ is a monoid of $\mathbf{F}$. Let us factorize $\pi$ as a composition of irreducible morphisms

$$
M=M_{r} \stackrel{\pi_{r}}{\longrightarrow} M_{r-1} \cdots M_{1} \stackrel{\pi_{1}}{\longrightarrow} M_{0}=N
$$

By Proposition 3.11, each $\pi_{i}$ is a $\ell \mathbf{A b}(p)$-morphism. We now prove by induction on $i$ that every language recognised by $M_{i}$ belongs to $\operatorname{Pol}_{p}\left(\mathcal{F}\left(A^{*}\right)\right)$. For $i=1$, the result follows from Theorem [5.9, since $\pi_{1}$ is a surjective $\ell \mathbf{A b}(p)$ morphism and since $N \in \mathbf{F}$. Suppose by induction that the result holds for $M_{i}$. Then by Theorem [5.9, every language fully recognised by $M_{i+1}$ belongs to $\operatorname{Pol}_{p}\left(\operatorname{Pol}_{p}\left(\mathcal{F}\left(A^{*}\right)\right)\right)$, which is equal to $\operatorname{Pol}_{p}\left(\mathcal{F}\left(A^{*}\right)\right)$ by Theorem 5.1, This proves the theorem since $\operatorname{Pol}_{p}\left(\mathcal{F}\left(A^{*}\right)\right.$ is contained in $\mathcal{W}_{2}\left(A^{*}\right)$.

The simplest instance of Theorem 6.2 is obtained by taking for $\mathbf{F}$ the trivial formation of monoids. In that case, one has $\mathcal{F}\left(A^{*}\right)=A^{*}$ for each alphabet $A$. Then $\mathbb{L} \mathbf{G}_{p} \mathbb{M} \mathbf{F}=\mathbf{G}_{p}$ and Theorem 6.2 states that, for each alphabet $A$, the formation of languages associated with $\mathbf{G}_{p}$ is the Boolean algebra generated by $\left(A^{*} a_{1} A^{*} \cdots a_{k} A^{*}\right)_{r, p}$, where $0 \leqslant r<p, k \geqslant 0$ and $a_{1}, \ldots, a_{k} \in A$ and we obtain Weil's result [30, 31, 32].

Taking for $\mathbf{F}$ the formation $\mathbf{Z}$, Propositions $4.2,3.15$ and Theorem 6.2 give immediately:

Proposition 6.3 Let $\mathcal{W}$ be the formation of languages corresponding to $\mathbb{I L} \mathbf{G}_{p}$. Then for each alphabet $A, \mathcal{W}\left(A^{*}\right)$ is the Boolean algebra generated by the languages of the form $\left(L_{0} a_{1} L_{1} \cdots a_{k} L_{k}\right)_{r, p}$, where each $L_{i}$ is either nondense or co-nondense.

Theorem 6.2 works with any formation of groups. Consider for instance the formation $\mathbf{F}$ generated by $A_{5}$, the alternating group of degree 5 . By $[8$, II.2.13] $\mathbf{F}$ is known to be the class of all direct products of copies of $A_{5}$ and therefore $\mathbf{F}$ is not a variety. The corresponding formation of languages $\mathcal{F}$ was described in $[3$. Now, Theorem 6.2 allow us to describe all languages of the class $\mathbf{G}_{p} * \mathbf{F}$.

Theorem 6.2 is of special interest for saturated formations. Let $\mathbf{F}$ be a saturated formation of groups locally defined by a formation function $f$. As we have seen, the canonical local definition $F$ of $\mathbf{F}$ can be obtained by setting $F(p)=\mathbf{G}_{p} *(f(p) \cap \mathbf{F})$, for each prime $p$. Let $\mathcal{C}_{p}$ be the formation of languages associated with the formation of groups $f(p) \cap \mathbf{F}$.

Corollary 6.4 Let $\mathcal{F}$ be the formation of languages associated with $\mathbf{F}$. For each alphabet $A, \mathcal{F}\left(A^{*}\right)$ is the Boolean algebra generated by the languages of the form $\left(L_{0} a_{1} L_{1} \cdots a_{k} L_{k}\right)_{r, p}$, where $L_{i} \in \mathcal{C}_{p}\left(A^{*}\right), 0 \leqslant i \leqslant k, 0 \leqslant r<p$ and $p$ runs over all primes. 
The precedent result shows that in order to describe the languages associated with a saturated formation $\mathbf{F}$ it is enough to know a description of the languages associated to any local definition of $\mathbf{F}$. We are now going to apply this method to several examples of local formations of groups and recover in this way a number of known results.

Example 6.1 (Nilpotent groups) As we have seen, the formation $\mathbf{N}$ of nilpotent groups is locally defined by $f(p)=(1)$, for all primes $p$. Since the formation of languages corresponding to $f(p)=(1)$ is, for each alphabet $A, \mathcal{C}_{p}\left(A^{*}\right)=A^{*}$, Corollary 6.4 states that, for each alphabet $A$, the formation of languages associated with $\mathbf{N}$ is the Boolean algebra generated by $\left(A^{*} a_{1} A^{*} \cdots a_{k} A^{*}\right)_{r, p}$, where $a_{1}, \ldots, a_{k} \in A, 0 \leqslant r<p$ and $p$ runs over all primes. Thus, we obtain Weil's result [30, 31] (see also [9, 23, 27, 28, 11]).

Example 6.2 (Soluble groups) Given a formation of groups $\mathbf{F}$, the class $\mathbf{N} * \mathbf{F}$ is locally defined by $f(p)=\mathbf{F}$ for all primes $p[8$, IV, (3.4)]. Therefore, knowing the languages of a formation $\mathbf{F}$, Corollary 6.4 allows us to describe the languages of the class $\mathbf{N} * \mathbf{F}$. We can apply this result to obtain, in particular, a description of the languages associated with the class of all soluble groups, first given by Straubing [23, 24].

Let $\left(\mathcal{N}_{i}\right)_{i \geqslant 0}$ be the family of formations of languages defined, for each alphabet $A$, by $\mathcal{N}_{0}\left(A^{*}\right)=\left\{\emptyset, A^{*}\right\}$ and for $i \geqslant 1, \mathcal{N}_{i}\left(A^{*}\right)$ is the Boolean algebra generated by the languages $\left(L_{0} a_{1} L_{1} \cdots a_{k} L_{k}\right)_{r, p}$, where $a_{1}, \ldots, a_{k} \in$ $A, 0 \leqslant r<p, p$ runs over all primes and each $L_{j} \in \mathcal{N}_{i-1}\left(A^{*}\right)$.

First, we know that the formation of languages associated with $\mathbf{N}$ is, for each alphabet $A, \mathcal{N}_{1}\left(A^{*}\right)$ (Example 6.1). Thus, by Corollary 6.4, the formation of languages associated with $\mathbf{N}^{2}=\mathbf{N} * \mathbf{N}$, is $\mathcal{N}_{2}\left(A^{*}\right)$ and, in general, the formation of languages corresponding to $\mathbf{N}^{i}=\mathbf{N} * \cdots * \mathbf{N}$ (the saturated formation of soluble groups with nilpotent length at most $i \geqslant 1$ ) is $\mathcal{N}_{i}\left(A^{*}\right)$.

Since the variety $\mathbf{S}$ of all soluble groups is the join of the varieties $\mathbf{N}^{i}$ for all integers $i \geqslant 0$, we deduce that the variety of languages corresponding to $\mathbf{S}$ is the join of the language varieties $\mathcal{N}_{i}$, for $i \geqslant 0$.

More generally, let $\pi$ be a set of primes and let $\mathbf{S}_{\pi}$ the variety of all soluble groups which orders are divisible only by primes in $\pi$. Let also $\left(\mathcal{N}_{\pi, i}\right)_{i \geqslant 0}$ denote the family of formations of languages defined as $\left(\mathcal{N}_{i}\right)_{i \geqslant 0}$ but considering only primes $p \in \pi$. The variety of languages $\mathcal{S}_{\pi}$ corresponding to $\mathbf{S}_{\pi}$ is the join of the language varieties $\left(\mathcal{N}_{\pi, i}\right)_{i \geqslant 0}$.

Example 6.3 (Supersoluble groups) The formation (or variety) of supersoluble groups is locally defined by $f(p)=\mathbf{A} \mathbf{b}(p-1)$, for all primes $p$, [6], [8, IV, (3.4)]. The formation of languages corresponding to $\mathbf{A b}(n)$ was described in [9] and, for each alphabet $A$, it is the Boolean algebra generated by the languages of the form $F(a, s, n)=\left\{\left.u \in A^{*}|| u\right|_{a} \equiv s \bmod n\right\}$, 
where $a \in A$ and $0 \leqslant s<n$. Thus, Corollary 6.4 states that the formation of languages associated with the variety of supersoluble groups is, for each alphabet $A$, the Boolean algebra generated by $\left(L_{0} a_{1} L_{1} \cdots a_{k} L_{k}\right)_{r, p}$, where $a_{1}, \ldots, a_{k}$ are letters of $A, 0 \leqslant r<p, p$ runs over all primes and each $L_{i}$ is a language of the form $F(a, s, p-1)$, for some $a \in A$ and $0 \leqslant s<p-1$, as it was obtained in [7].

Example 6.4 (Sylow tower groups of type $\prec$ ) Let $\prec$ be an arbitrary linear ordering on the set $\mathbb{P}$ of all primes. Let $G$ be a group such that $|G|=p_{1}^{n_{1}} \ldots p_{r}^{n_{r}}$, with $p_{1}, \ldots, p_{r}$ primes such that $p_{1} \prec p_{2} \prec \cdots \prec p_{r-1} \prec p_{r}$. We say that $G$ has a Sylow tower of type $\prec$ if $G \in \mathbf{G}_{p_{1}} * \mathbf{G}_{p_{2}} * \cdots * \mathbf{G}_{p_{r}}$. The class $\mathbf{T}_{\prec}$ of all soluble groups with a Sylow tower of type $\prec$ is a saturated formation. Given a prime $p$, let $\pi(p)=\{q \in \mathbb{P} \mid p \prec q\}$ and put $f(p)=\mathbf{S}_{\pi(p)}$. It follows that $\mathbf{T}_{\prec}$ is locally defined by $f[8, \mathrm{IV},(3.4)]$. Consequently, by Corollary 6.4, the formation of languages associated whith $\mathbf{T}_{\prec}$ is, for each alphabet $A$, the Boolean algebra generated by $\left(L_{0} a_{1} L_{1} \cdots a_{k} L_{k}\right)_{r, p}$, where $a_{1}, \ldots, a_{k}$ are letters of $A, 0 \leqslant r<p, p$ runs over all primes and each $L_{i}$ is a language of $\mathcal{S}_{\pi(p)}$.

Example 6.5 (Fitting varieties of soluble groups) A Fitting variety is a variety $\mathbf{F}$ which is closed by the following property: whenever a group $G$ is generated by subnormal subgroups $N_{1}, \ldots, N_{r} \in \mathbf{F}$ then $G \in \mathbf{F}$. Whereas $\mathbf{N}$ and $\mathbf{S}$ are examples of Fitting varieties, the classes of abelian groups and of supersoluble groups are not. In general, Fitting varieties are not saturated. In fact, in [2] Ezquerro and the first author characterised the Fitting varieties of groups which are saturated. Nevertheless, in the soluble universe a Fitting variety is always saturated [5], 4] and, therefore, it can be defined locally. If $F$ is the canonical local definition of a Fitting variety $\mathbf{F}$ of soluble groups, then $F(p)$ is again a Fitting variety for all primes $p$ [8, IV, (3.16)]. In particular, $F(p)$ is saturated and can be defined locally for all primes $p$. Since the languages of $\mathbf{S}_{\pi}$ are known for any set $\pi$ of primes, our results give a way to construct the class of languages associated to any Fitting variety of soluble groups.

\section{References}

[1] J. Almeida, S. Margolis, B. Steinberg and M. Volkov, Representation theory of finite semigroups, semigroup radicals and formal language theory, Trans. Amer. Math. Soc. 361,3 (2009), 1429-1461.

[2] A. Ballester-Bolinches And L. M. Ezquerro, On a theorem of Bryce and Cossey, Bull. Austral. Math. Soc. 57,3 (1998), 455-460.

[3] A. Ballester-Bolinches, J.-E. Pin and X. Soler-Escrivà, Formations of finite monoids and formal languages: Eilenberg's variety 
theorem revisited, Forum Mathematicum ISSN (Online) 1435-5337, ISSN (Print) 0933-7741, DOI: 10.1515/forum-2012-0055, June 2012 (2012). To appear in Forum Mathematicum.

[4] R. A. Bryce and J. Cossey, Corrigenda: "Subgroup closed Fitting classes" [Math. Proc. Cambridge Philos. Soc. 83 (1978), no. 2, 195-204; MR 80g:20031], Math. Proc. Cambridge Philos. Soc. 91,2 (1982), 343.

[5] R. A. Bryce and J. Cossey, Subgroup closed Fitting classes are formations, Math. Proc. Cambridge Philos. Soc. 91,2 (1982), 225-258.

[6] R. Carter and T. Hawkes, The $\mathcal{F}$-normalizers of a finite soluble group, J. Algebra 5 (1967), 175-202.

[7] O. Carton, J.-E. Pin and X. Soler-Escrivà, Languages Recognized by Finite Supersoluble Groups, Journal of Automata, Languages and Combinatorics 14,2 (2009), 149-161.

[8] K. Doerk and T. Hawkes, Finite soluble groups, de Gruyter Expositions in Mathematics vol. 4, Walter De Gruyter \& Co., Berlin, 1992.

[9] S. Eilenberg, Automata, languages, and machines. Vol. B, Academic Press [Harcourt Brace Jovanovich Publishers], New York, 1976. Pure and Applied Mathematics, Vol. 59.

[10] P. PÉladeau, Sur le produit avec compteur modulo un nombre premier, RAIRO Inform. Théor. Appl. 26,6 (1992), 553-564.

[11] P. PÉladeau and D. Thérien, Sur les langages reconnus par des groupes nilpotents, C. R. Acad. Sci. Paris Sér. I Math. 306,2 (1988), 93-95.

[12] J.-É. Pin, Finite group topology and $p$-adic topology for free monoids, in 12th ICALP, W. Brauer (ed.), pp. 445-455, Lect. Notes Comp. Sci. vol. 194, Springer, 1985.

[13] J.-É. PIN, Varieties of formal languages, North Oxford, London et Plenum, New-York, 1986. (Translation of Variétés de langages formels).

[14] J.-É. PIN, Topologies for the free monoid, J. of Algebra 137 (1991), 297-337.

[15] J.-É. Pin, H. Straubing And D. ThéRIEn, Locally trivial categories and unambiguous concatenation, J. Pure Appl. Algebra 52 (1988), 297311.

[16] J.-É. Pin And P. WeIL, Profinite semigroups, Mal'cev products and identities, J. of Algebra 182 (1996), 604-626. 
[17] J. Rhodes, A homomorphism theorem for finite semigroups, Math. Systems Theory 1 (1967), 289-304.

[18] J. Rhodes And B. Steinberg, The q-theory of finite semigroups, Springer Monographs in Mathematics, Springer, New York, 2009.

[19] J. Rhodes And B. Tilson, The kernel of monoid morphisms, J. Pure Appl. Algebra 62,3 (1989), 227-268.

[20] J. Rhodes and P. Weil, Decomposition techniques for finite semigroups, using categories. I, II, J. Pure Appl. Algebra 62,3 (1989), 269$284,285-312$.

[21] L. A. Shemetkov And A. N. SkibA, Formations of algebraic systems. (Formatsii algebraicheskikh sistem.), Современная Алгебра. [Modern Algebra], Sovremennaya Algebra. Moskva: Nauka. 256 P. R. 3.00, Moscow, 1989. With an English summary.

[22] I. Simon, Hierarchies of Events with Dot-Depth One, PhD thesis, University of Waterloo, Waterloo, Ontario, Canada, 1972.

[23] H. Straubing, Varieties of Recognizable Sets Whose Syntactic Monoids Contain Solvable Groups, $\mathrm{PhD}$ thesis, University of California, Berkeley, CA, 1978.

[24] H. Straubing, Families of recognizable sets corresponding to certain varieties of finite monoids, J. Pure Appl. Algebra 15,3 (1979), 305-318.

[25] H. Straubing, Relational morphisms and operations on recognizable sets, RAIRO Inf. Theor. 15 (1981), 149-159.

[26] H. Straubing, Finite semigroup varieties of the form $V * D$, J. Pure Appl. Algebra 36,1 (1985), 53-94.

[27] D. ThÉRIEn, Languages of Nilpotent and Solvable Groups (Extended Abstract), in ICALP, H. A. Maurer (ed.), pp. 616-632, Lect. Notes Comp. Sci. vol. 71, Springer, 1979.

[28] D. Thérien, Subword counting and nilpotent groups, in Combinatorics on words (Waterloo, Ont., 1982), pp. 297-305, Academic Press, Toronto, ON, 1983.

[29] D. ThÉrien, On the equation $x^{t}=x^{t+q}$ in categories, Semigroup Forum 37,3 (1988), 265-271.

[30] P. WeIL, An extension of the Schützenberger product, in Lattices, semigroups, and universal algebra (Lisbon, 1988), pp. 315-321, Plenum, New York, 1990. 
[31] P. WeIL, Products of languages with counter, Theoret. Comput. Sci. 76 (1990), 251-260.

[32] P. WeIL, Closure of varieties of languages under products with counter, J. Comput. System Sci. 45 (1992), 316-339. 